
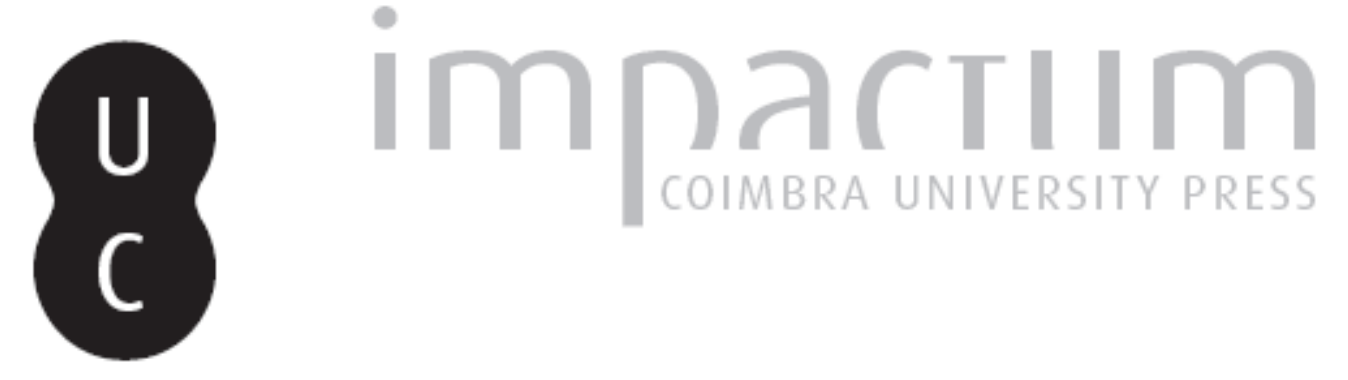

\title{
Cambios de uso y erosión de suelos: análisis diacrónico (1960-2012) mediante SIG en la cuenca del Guiniguada (Gran Canaria, Islas Canarias, España)
} \author{
$\begin{array}{ll}\text { Autor(es): } & \text { Romero Martín, Lidia Esther; García Romero, Leví; Hernández Cordero, } \\ \text { Antonio; Pérez-Chacón Espino, Emma }\end{array}$ \\ Publicado por: $\quad$ Associação Portuguesa de Riscos, Prevenção e Segurança; Imprensa \\ da Universidade de Coimbra \\ URL \\ persistente: URI:http://hdl.handle.net/10316.2/41212 \\ DOI: ～DOI:https://doi.org/10.14195/1647-7723_24_5
}

Accessed : $\quad$ 26-Apr-2023 14:24:51

A navegação consulta e descarregamento dos títulos inseridos nas Bibliotecas Digitais UC Digitalis, UC Pombalina e UC Impactum, pressupõem a aceitação plena e sem reservas dos Termos e Condições de Uso destas Bibliotecas Digitais, disponíveis em https://digitalis.uc.pt/pt-pt/termos.

Conforme exposto nos referidos Termos e Condições de Uso, o descarregamento de títulos de acesso restrito requer uma licença válida de autorização devendo o utilizador aceder ao(s) documento(s) a partir de um endereço de IP da instituição detentora da supramencionada licença.

Ao utilizador é apenas permitido o descarregamento para uso pessoal, pelo que o emprego do(s) título(s) descarregado(s) para outro fim, designadamente comercial, carece de autorização do respetivo autor ou editor da obra.

Na medida em que todas as obras da UC Digitalis se encontram protegidas pelo Código do Direito de Autor e Direitos Conexos e demais legislação aplicável, toda a cópia, parcial ou total, deste documento, nos casos em que é legalmente admitida, deverá conter ou fazer-se acompanhar por este aviso.

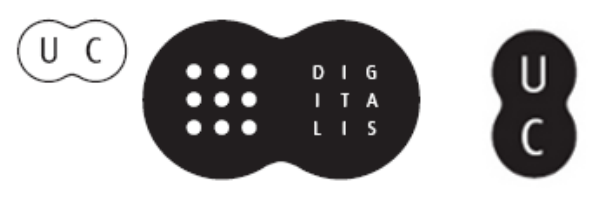




\section{MULTIDISCIPLINARIDADE NA ANÁLISE DAS MANIFESTAÇÕES DE RISCO}

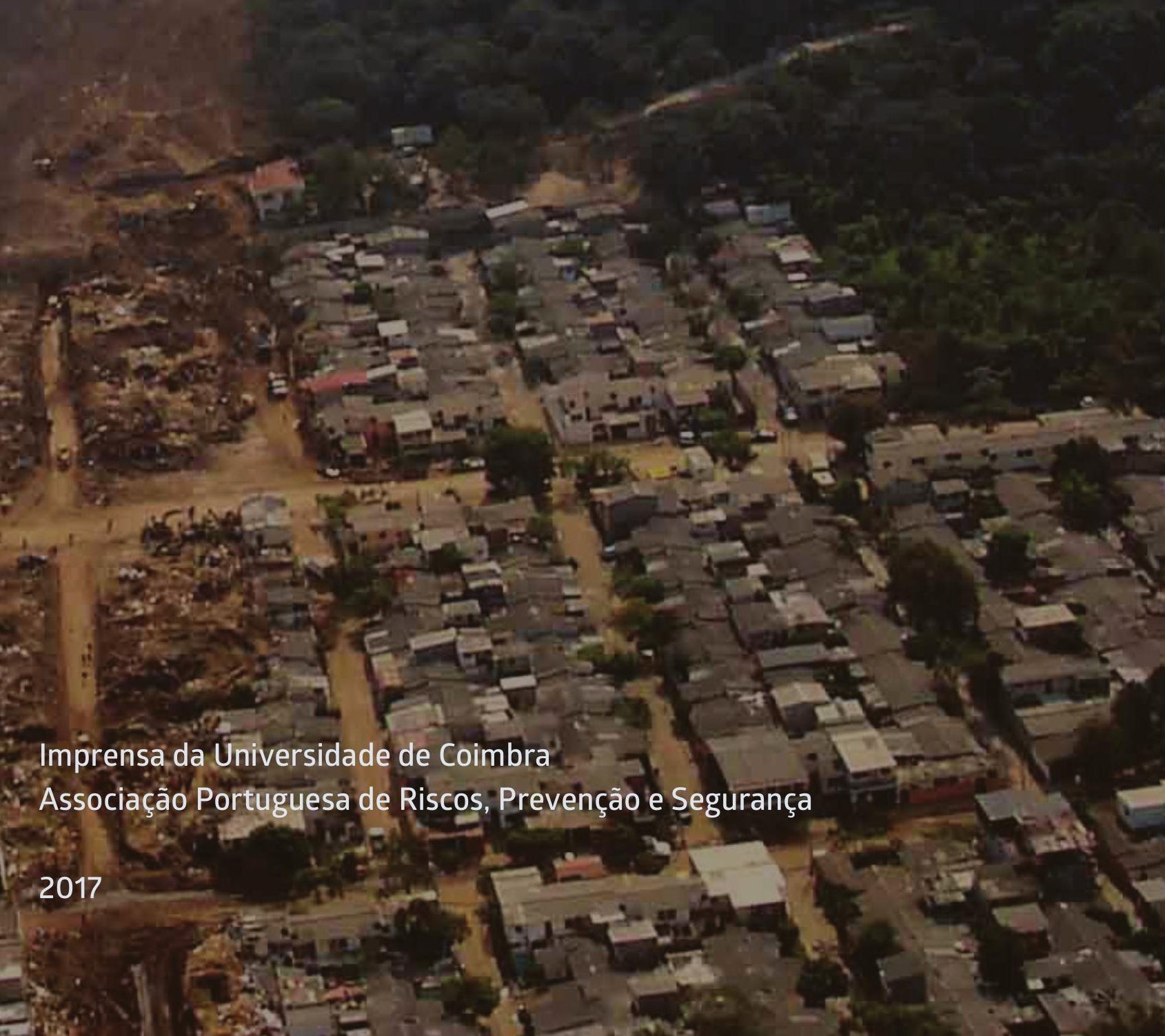




\section{CAMBIOS DE USO Y EROSIÓN DE SUELOS:ANÁLISIS DIACRÓNICO (1960-2012) MEDIANTE SIG EN LA CUENCA DEL GUINIGUADA (GRAN CANARIA, ISLAS CANARIAS, ESPAÑA)*}

CHANGES IN USE AND SOIL EROSION: DIACHRONIC ANALYSIS (1960-2012) GIS IN GUINIGUADA BASIN (GRAN CANARIA, CANARY ISLANDS, SPAIN)

Lidia Esther Romero Martín Instituto de Oceanografía y Cambio Global (IOCAG), Universidad de Las Palmas de Gran Canaria lidia.romero@ulpgc.es

Leví García Romero Instituto de Oceanografía y Cambio Global (IOCAG), Universidad de Las Palmas de Gran Canaria levi.garcia@ulpgc.es

Antonio Hernández Cordero Instituto de Oceanografía y Cambio Global (IOCAG), Universidad de Las Palmas de Gran Canaria hernandez.cordero@ulpgc.es

Emma Pérez-Chacón Espino Instituto de Oceanografía y Cambio Global (IOCAG), Universidad de Las Palmas de Gran Canaria emma.perez-chacon@ulpgc.es

\section{RESUMEN}

Se analiza la evolución de las tasas de erosión tras los cambios de usos del suelo producidos entre 1960 y 2012 . Para ello se adapta el modelo empírico USLE a las especificidades de Gran Canaria, y se implementa mediante un SIG. Los resultados muestran que la eficacia antierosiva y la calidad paisajística, de las superficies abancaladas de la cuenca, se han visto desigualmente afectadas por el incremento de las tasas de erosión.

Palabras clave: Erosión, cambios de coberturas del suelo, SIG, cuenca, terrazas agrícolas.

\section{ABSTRACT}

The evolution of the erosion rates in the Guiniguada ravine (Gran Canaria, Spain), between 1960 and 2012, is here analyzed. For the study, the empirical model USLE is applied, adapting it to the specificities of the Gran Canaria, and the analysis are developed using GIS. The results show that the anti-erosion efficiency and landscape quality of terraced surfaces in the study area have been unevenly affected by the increase of erosion rates.

Keywords: Erosion, land cover changes, GIS, watershed, agricultural terraces.

\section{RESUMO}

Mudanças no uso e erosão dos solos: análise diacrónica (1960-2012) GIS na Bacia do Guiniguada (Gran Canaria, Ilhas Canárias, Espanha) - A evolução das taxas de erosão é analisada após as mudanças no uso do solo produzidas entre 1960 e 2012. Para esse feito, recorreu-se ao modelo empírico USLE, adaptado às especificidades de Gran Canaria e implementado através de um SIG. Os resultados mostram que a eficácia anti-erosão e a qualidade da paisagem, bem como a superfície dos terraços da bacia foram afetados de forma desigual com o aumento das taxas de erosão.

Palavras-chave: Erosão, mudanças do uso do solo, GIS, Bacias Hidrográficas, terraços agrícolas.

\section{RESUMÉ}

Changements d'occupation et érosion des sols: analyse diachronique (1960-2012) SIG dans le bassin versant Guiniguada (Gran Canaria, Îles Canaries, Espagne) - Nous avons analysé l'évolution des taux d'érosion après les changements d'utilisation du sol entre 1960 et 2012. Pour cela, nous avons adapté le modèle empirique USLE aux spécificités du bassin versant de Gran Canaria et nous l'avons mis en œuvre à travers un SIG. Les résultats montrent que l'efficacité antiérosive et la qualité paysagère des terrasse dans le bassin ont été atteintes de façon inégale par une augmentation des taux d'érosion.

Mots-clé: Érosion, changements de couverture du sol, SIG, bassin versant, terrasses agricoles.

* O texto deste artigo corresponde a uma comunicação apresentada no I Seminário da Rede Incêndios-Solo e I Simpósio Ibero-Afro-Americano de Riscos, tendo sido submetido em 16-12-2015 sujeito a revisão por pares a 09-01-2016 e aceite para publicação em 18-04-2016.

Este artigo é parte integrante da Revista Territorium, $n .{ }^{\circ} 24,2017,{ }^{\circ} \mathrm{RIscos}$, ISSN: 0872-8941. 


\section{Introduction}

El suelo es un recurso natural con un valor ecológico notable, pues no sólo es soporte de la vegetación y de la fauna, almacén de agua y nutrientes y regulador hidrológico, sino que también representa el sustento de la alimentación humana. Además, en los suelos agrícolas, a su valor económico se suma su elevado valor cultural, pues sintetiza un importante legado histórico en la gestión de recursos hídricos y edáficos En islas y en áreas de montaña, alejadas de los grandes centros de producción de alimentos, éste debe ser considerado también "patrimonio estratégico de supervivencia" (Rodríguez-Rodríguez, 2002:320).

Entre los problemas medioambientales que presenta el planeta Tierra, la erosión es el proceso que tiene mayor incidencia en la degradación del suelo. La erosión constituye una importante amenaza ambiental para la sostenibilidad y la capacidad productiva de la agricultura. Los procesos de erosión, además de la pérdida de suelo agrícola y forestal, también reducen la calidad del suelo (fertilidad) que permanece en cultivo. La erosión ha sido la causante de la pérdida de casi un tercio de las tierras cultivables del mundo, cuestión que prosigue a un ritmo de más de 10 millones de hectáreas por año (Yang et al., 2003).

El problema de la erosión es especialmente grave en el área mediterránea de Europa y en las Islas Canarias. El $43 \%$ del territorio canario experimenta una erosión hídrica y eólica acelerada grave, es decir, superior a $12 \mathrm{t} / \mathrm{ha} / \mathrm{año}$, que equivale a una pérdida de 1-1,5 mm anuales, considerado como el valor límite tolerable de erosión (Schmidt et al., 1982). En el caso de Gran Canaria esa magnitud afecta al $56,7 \%$ de su superficie insular (Fernández- Palacios et al.,2004:98).

El tipoy el grado de intervención humana sobre el territorio afectan de manera diferente a las características de los suelos (estabilidad, procesos hidrológicos), a la cubierta vegetal e incluso a la topografía de las vertientes (construcción de bancales o de sistemas de drenaje de la escorrentía) y, en consecuencia, a la erosión del suelo. Por ello, cambios en los usos del suelo suponen, a su vez, modificaciones en la dinámica hidrológica y geomorfológica del territorio. Por su parte, el abandono de la agricultura es uno de los cambios de uso del suelo más extendido en los países desarrollados, especialmente en los de la ribera del Mediterráneo desde mediados del siglo XX. En las Islas Canarias, la magnitud e intensidad de los cambios que han experimentado los paisajes agrícolas durante los últimos cincuenta años no tiene precedentes en su historia.

El abandono de las prácticas agrarias no siempre supone la regeneración de los ecosistemas destruidos en el pasado. Por ejemplo, en las vertientes abancaladas, donde la energía utilizada por la sociedad para su construcción ha sido muy elevada, también hace falta mucha energía para mantenerlas; y la vegetación, por si sola, tiene dificultades para evitar los movimientos en masa que se instalan en los bordes de las terrazas (García-Ruiz et al., 1988). Muchos autores señalan que el proceso de colonización vegetal tras el abandono, junto a la caída de los muros de los bancales (Jongman, 2002; Agnoletti, 2006 y 2007; Errea et al., 2009 y Lasanta, 2010) conllevan un incremento del riesgo de génesis y propagación de incendios, así como la pérdida de la calidad estética y del valor cultural del paisaje. Todo ello reduce la capacidad de atracción turística que podrían tener estos espacios.

El estudio de este problema ambiental y social se puede abordar a partir de diferentes métodos, según los objetivos propuestos y las escalas temporal y espacial seleccionadas. En este trabajo, el objetivo general es analizar la evolución experimentada por las tasas de erosión hídrica superficial tras los cambios de usos del suelo de los últimos 52 años (1960-2012). El área de estudio es una cuenca hidrográfica situada en el noreste de la isla de Gran Canaria (Islas Canarias, España). Los objetivos específicos son los siguientes: en primer lugar, valorar los cambios (incremento o decremento) de las tasas de erosión, en términos de balance, durante el periodo temporal considerado; $y$, en segundo lugar, analizar la distribución espacial de esas tasas para identificar las áreas que presentan actualmente una mayor vulnerabilidad erosiva.

El procedimiento de valoración seleccionado se sustenta en el modelo empírico y paramétrico USLE, Ecuación Universal de Pérdida de Suelos (Wischmeier y Smith, 1978). Resulta el más adecuado por su carácter sintético, facilidad de cálculo y aplicabilidad territorial y espacio-temporal. Las técnicas empleadas son la fotointerpretación, la geoestadística y los Sistemas de Información Geográfica (SIG). La interpretación de fotogramas aéreos y de ortofotos permite obtener información sobre los usos, coberturas y prácticas de conservación de suelos (aterrazamientos). Y la geoestadística junto con los Sistemas de Información Geográfica son las herramientas empleadas para la implementación del modelo erosivo escogido (Dumas, 2012). Estas técnicas permiten procesar un volumen importante

de datos; calcular algunas de las variables implicadas en el modelo; obtener capas para cada factor de la ecuación matemática; y ejecutar operaciones y análisis multicapas. A su vez, estas herramientas permiten conocer la distribución espacial de los factores y de los resultados obtenidos, al tiempo que facilitan su visualización cartográfica.

El modelo erosivo USLE ha sido aplicado en diferentes partes del mundo, tanto en su versión original, como en las de las sucesivas modificaciones (RUSLE - K.G. Renard, 
et al., 1991, MUSLE - I.R. Williams, 1975 y RUSLE3D - H. Mitasova et al., 1996) que ha experimentado.

A escala global, y relacionando los cambios de uso del pasado con los escenarios futuros de cambios de uso y de cambio climático, destaca el trabajo de Yang et al. (2003). En América, concretamente en USA, son muchos los trabajos realizados, entre los que destacan el de Martin et al. (2003). En el centro y sur de América sobresalen los realizados en México (Montes el al., 2001; Flores et al., 2003), Puerto Rico (Rojas-González, 2008), la Amazonia brasileña (Lu et al., 2004), Chile (Araneda, 2002) y Argentina (Gaspari et al., 2009a y b; Orúe et al., 2007). En el continente asiático destacan los trabajos realizados en China (Ma, et al., 2003,), Corea (Lee, 2004) y La India (Pandey et al., 2007; Jain, M.K. y Das, D., 2010). En África, concretamente en Marruecos, es reseñable el trabajo de Lahlaoi et al. (2015). Finalmente, en Europa, cabe destacar el cálculo de la erosión para todo el continente, realizado por Van der Knijff et al. (2000) y en cada uno de los países siguientes: Alemania (Jäger, 1994), Bélgica (Van Rompaey et al., 2000), Italia (Grimm et al. 2003), Turquía (Erdogan et al. 2007 y Ozcan et al., 2008), Grecia-Creta (Kouli et al., 2009) y Portugal (Panagapoulos et al., 2011 y Reis et al., 2014). En España se está realizando el Inventario Nacional de Erosión de Suelos (INES 2002-2012) (Torres-Quevedo et al., 2004; Delgado y Del Palacio, 2005) y se ha aplicado a escala de cuencas hidrográficas en Madrid (De Antonio, 1994), Badajoz (Rodríguez et al., 2004), Andalucía (Geler et al., 2004), Guadalajara (Arévalo et al., 2011) y Ávila (Hernández-Rodríguez et al., 2014).

En las Islas Canarias, dicho modelo se aplicó para el cálculo de la erosión actual y la potencial de la isla de Gran Canaria, cuyos mapas forman parte una serie cartográfica (Sánchez et al., 1995) que proporciona una base científica para la planificación ambiental y la ordenación del territorio. También se emplea el modelo USLE para el cálculo de erosión que se genera con la extracción y traslado de suelo vegetal desde las medianías (zonas situadas entre los 600 y 1000 metros de altitud) del norte de Gran Canaria hacia las superficies agrícolas de la costa (sorriba) (Hernández-Calvento, 1996 y Hernández-Calvento et al., 1998). También se ha utilizado para comparar la evolución de las tasas de erosión en dos cuencas hidrográficas con características ambientales y de aprovechamiento antrópico diferentes, en dos momentos de la historia socioeconómica reciente (1960-2002) (Abadín et al., 2004 y Abadín, 2007).

\section{Área de estudio}

La zona seleccionada para este estudio es la cuenca del Guiniguada, localizada en el noreste de la isla de Gran Canaria (Islas Canarias, España) y con una superficie de $65 \mathrm{~km}^{2}$ (fig. 1). Su ubicación en el sector litológicamente más joven de esa isla volcánica determina el predominio de materiales volcánicos pliocuaternarios (coladas basálticas, brechas y superficies piroclásticas) y de morfologías juveniles. Estas son formas volcánicas directas y barrancos encajados separados por interfluvios alomados. Presenta una disposición NE-SO y el relieve asciende, de forma general, de NE a SE, donde se localiza su punto más elevado (Cruz del Saucillo, a 1.800 m.).

Las pendientes moderadas (entre $10^{\circ}$ y $25^{\circ}$ ) y las acusadas $\left(>25^{\circ}\right)$ ocupan una superficie similar: el $39 \%$ el 36,7\% del área de la cuenca, respectivamente. Las primeras conforman las vertientes de los interfluvios alomados, de los conos volcánicos, así como buena parte de la divisoria topográfica de la cuenca y, las segundas, se localizan en los taludes de derrubios, en las paredes de domos fonolíticos de las cabeceras meridionales de la cuenca, así como en escarpes y laderas acentuadas.

Las vertientes rectilíneas, que poseen suelos poco potentes y pendientes pronunciadas, ocupan el $54,1 \%$ del total de la superficie de la cuenca siendo exportadoras de agua, suelo y nutrientes.

La cuenca presenta las características climáticas propias de los sectores de barlovento de Canarias. Es decir, se encuentra regularmente afectada por las masas de aire húmedas de componente NE (derivadas de los vientos alisios), de componente NO (perturbaciones atlánticas) $y$, en menor medida, de SO (borrascas atlánticas con recorrido meridional). Su exposición Norte y el carácter masivo de su relieve (desnivel de 1.800 metros en apenas $22 \mathrm{~km}$, de costa a cumbre) explica la gradación de las

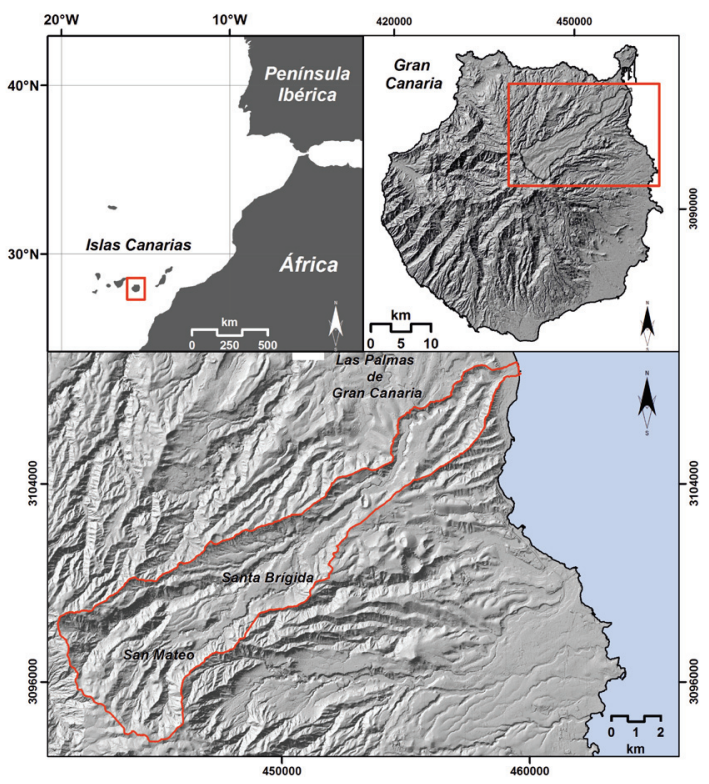

Fig. 1 - Localización del área de estudio. Cuenca del Guiniguada (Islas Canarias. España).

Fig. 1 - Location of the study area. Guiniguada Basin (Canary Islands. Spain). 
características bioclimáticas en cuatro geoambientes: costa, medianías bajas, medianías altas y cumbres. Los registros termopluviométricos medios anuales varían desde los $21^{\circ} \mathrm{C}$ y $140,4 \mathrm{~mm}$ en la costa hasta los $14,3^{\circ} \mathrm{C}$ y más de $800 \mathrm{~mm}$ en la cumbre.

Los suelos presentan una clara diferenciación altitudinal, condicionada por la litología y el clima. En la costa, bajo condiciones subdesérticas y sobre materiales sedimentarios, se encuentran los calcisoles y vertisoles. En medianías, sobre materiales jóvenes, en ambientes subhúmedos y húmedos y sobre laderas de pendientes acusadas, destacan los cambioles, los luvisoles y los leptosoles respectivamente. $Y$ en el geoambiente de cumbre, sobre materiales piroclásticos jóvenes, se desarrollan suelos jóvenesminerales, los andosoles.

El paisaje vegetal de esta cuenca muestra claros signos de la intensa antropización experimentada a lo largo de los últimos cinco siglos. Prueba de ello es que el 46,84 $\%$ de su superficie carece de cobertura vegetal natural (mapa de vegetación de Gran Canaria, GRAFCAN, 2006). De ella, tan sólo el 29,17 \% está actualmente destinado a la actividad agrícola, mientras que el $17,60 \%$ lo ocupan las áreas edificadas. Por otra parte, el paisaje vegetal dominante es el de la vegetación de sustitución (40\%), cuyas comunidades colonizan el terreno tras el abandono de las actividades agrarias tradicionales (agricultura, pastoreo, aprovechamiento forestal). Tan sólo quedan pequeños enclaves en los que se conservan vestigios del paisaje vegetal natural. Estos relictos de vegetación suponen pequeñas islas de biodiversidad genética y ocupan apenas el $12,79 \%$ de la superficie de la cuenca.

En la actualidad residen en este espacio 42.865 personas (INE, 2011), presenta una densidad demográfica de 659,5 habitantes $/ \mathrm{km}^{2}$. Durante el periodo temporal abarcado en este trabajo la población de la cuenca se ha duplicado, pues en 1960 contaba con tan sólo 24.587 habitantes. Pero ese crecimiento no es generalizado en toda el área de estudio, contrastando la dinámica progresiva de las entidades de población situadas en la desembocadura (capital insular), frente a la dinámica regresiva de las que se encuentran en el interior, especialmente en la cabecera de la cuenca.

El paisaje agrícola de la cuenca del Guiniguada comparte la principal característica de las vertientes de barlovento de las islas más montañosas de Canarias: su elevada agrodiversidad. Las huertas y las patatas son los cultivos dominantes. Se aprecia una clara gradación altitudinal en la variedad de cultivos leñosos: desde los frutales subtropicales de la costa a los cítricos de las medianías bajas y frutales templados de medianías altas y cumbre. El proceso de cambio reciente más destacado en este espacio es la desagrarización. Prueba de ello es que el 67,62\% de su superficie presenta en 2005 (mapa de cultivos de Gran Canaria, Gobierno de Canarias, 2005) usos no agrícolas (eriales, monte, viales y espacios construidos). Se suma a ese dato el reducido número de personas que se dedican, como actividad lucrativa, al sector primario que supone tan sólo el 1,8 \% de su población activa.

La cuenca del Guiniguada ha experimentado pues una profunda transformación, aunque aún se pueden observar abundantes huellas de su pasado agrícola, como la extensa superficie abancalada que presenta, y que abarca todos los geoambientes de la cuenca (Romero, 2015). Ante esta situación, el patrimonio abancalado se muestra actualmente más vulnerable que nunca ante los procesos de erosión. El tipo de agricultura que impera en la actualidad, a "tiempo parcial", no garantiza el mantenimiento de estas estructuras agrícolas, ni la pervivencia de estos paisajes culturales, o la soberanía alimentaria y la conservación de los escasos suelos agrícolas que existen (Romero et al., 2015).

\section{Metodología}

Como ya se ha señalado en párrafos anteriores, el método empleado para el cálculo de la erosión hídrica superficial en las dos fechas señaladas es la Ecuación Universal de Pérdida de Suelo (USLE). La erosión es un proceso complejo que resulta de la relación de factores físicos y socioeconómicos. Los factores físicos dependen de procesos naturales como la intensidad de las precipitaciones, la erodibilidad del suelo y la topografía (pendiente y longitud de la ladera). Y los factores socioeconómicos son los relacionados con los usos del suelo, la cobertura vegetal y la aplicación de prácticas de conservación del suelo.

La formulación original de la USLE se realiza de la siguiente manera:

$$
A=R \cdot K \cdot L S \cdot C \cdot P
$$

Donde,

$A$ = pérdida de suelo (t/ha/año)

$\boldsymbol{R}=$ Factor de erosividad de la lluvia (MJ/ha/año) $(\mathrm{mm} / \mathrm{h}) / 10$

$K=$ Factor de erodibilidad del suelo (t ha $\mathrm{h} / \mathrm{ha} M T \mathrm{~mm}$ )

$L S=$ Factor longitud-pendiente de la vertiente (adimensional)

C = Factor cobertura del suelo (adimensional)

$\boldsymbol{P}=$ Factor prácticas de conservación del suelo (adimensional)

A continuación, se expone el concepto utilizado para cada factor, las fuentes de información empleadas y los procedimientos seguidos para su cálculo. 


\section{FACTOR R}

La erosividad de la lluvia, o índice de erosión pluvial, recoge la influencia de la intensidad y el volumen de la precipitación en la erosión. El reducido número de pluviógrafos y de series temporales largas con datos de 130 (intensidad máxima de lluvia en 30 minutos) hace inviable la aplicación de la fórmula original. En su defecto, se trabaja con datos de lluvias medias mensuales y anuales (series de 50 años) de 25 estaciones pluviométricas, pertenecientes a la red de estaciones del Servicio Hidráulico de Las Palmas; lo que supone una densidad de 2,6 pluviómetros por $\mathrm{km}^{2}$. De ellas, ocho se encuentran fuera de los límites topográficos de la cuenca. Se han considerado porque en el proceso de interpolación es necesario contar con puntos muestrales en las áreas periféricas, pues de no ser así el sistema tiende a considerar un gradiente lineal, según la tendencia creciente o decreciente de los valores más cercanos.

El procedimiento seguido para su cálculo y representación cartográfica es el siguiente:

a) Análisis de la calidad de las series pluviométricas. Se comprobó que su longitud y homogeneidad fueran aceptables y se rellenaron las lagunas de datos (gaps) según método de correlación (Fernández García, 1995).

b) Cálculo de la erosividad pluviométrica $(R)$. Se sigue el procedimiento empleado por otros autores en las islas Canarias (Padrón et al., 1991; Sánchez et al., 1995; Hernández-Calvento, 1996 y HernándezCalvento et al., 2002), que consiste en aplicar el índice de Fournier modificado por Arnoldus (Fm) en 1980. Presenta la ventaja del uso de datos pluviométricos más accesibles y abundantes (la lluvia media mensual), obteniendo una buena correlación con el factor $\mathrm{R}$ de la USLE. La expresión de este índice es:

$$
\mathrm{Fm}=\sum_{\mathrm{i}=1}^{12} \frac{\mathrm{p}_{\mathrm{i}}^{2}}{\mathrm{P}}
$$

donde pi representa la precipitación mensual y $\mathrm{P}$ la precipitación anual.

En el caso de la isla de Gran Canaria, Sánchez et al. (1995) calculan este índice para las mismas estaciones pluviométricas de la isla de Gran Canaria en las que el ICONA calcula el factor $\boldsymbol{R}$ y, aplicando varias ecuaciones de regresión, observan que el mayor factor de correlación $\left(r^{2}=0,88\right)$ viene dado por la ecuación exponencial:

$$
R=\exp (3,11503+0,0119296 \cdot \mathrm{Fm})
$$

Esa es la fórmula que se aplica a las 25 estaciones pluviométricas del Guiniguada, para el cálculo de la $R$ en este trabajo.

c) Cálculo de la relación entre la erosividad media anual y la altitud, con el fin de conocer cómo esta última variable condiciona la distribución de ese factor pluviomético. Tras varios ensayos, la recta de regresión que presentó mejor ajuste fue de tipo exponencial (fig. 2).

d) Cálculo de la R' para las mismas altitudes a las que se localizan las estaciones pluviométricas para conocer la bondad de ajuste de dicha recta de regresión, de la que resulta un error medio cuadrático de 6,7 (MJ/ ha/año) $(\mathrm{mm} / \mathrm{h}) / 10$ y un error cuadrático máximo de 14,4 (MJ/ha/año) $(\mathrm{mm} / \mathrm{h}) / 10$ (TABLA I).

Comprobación estadística de la validez de los datos obtenidos con un test de significación de correlación, cuyo resultado $\left(R^{2}=0,9471\right)$ indica que ambas muestras son casi idénticas (fig. 2)

f) Elaboración del mapa del factor $\mathrm{R}$ a partir de la interpolación a toda la cuenca de la función de regresión obtenida, a toda la cuenca utilizando el MDE como variable independiente. Se obtiene un modelo digital cuyos valores $\mathrm{R}$ vienen representados de forma continua con resolución espacial de 5 metros.

\section{FACTOR K}

El factor $\mathrm{K}$ es un índice de susceptibilidad del suelo a la erosión. Representa la pérdida de suelo por unidad de índice de erosión pluvial. Es una función de cinco parámetros del suelo: porcentaje de limo + arena muy fina $(0,002-0,1 \mathrm{~mm})$, porcentaje de arena $(0,1-$ $0,2 \mathrm{~mm})$, porcentaje de materia orgánica, estructura

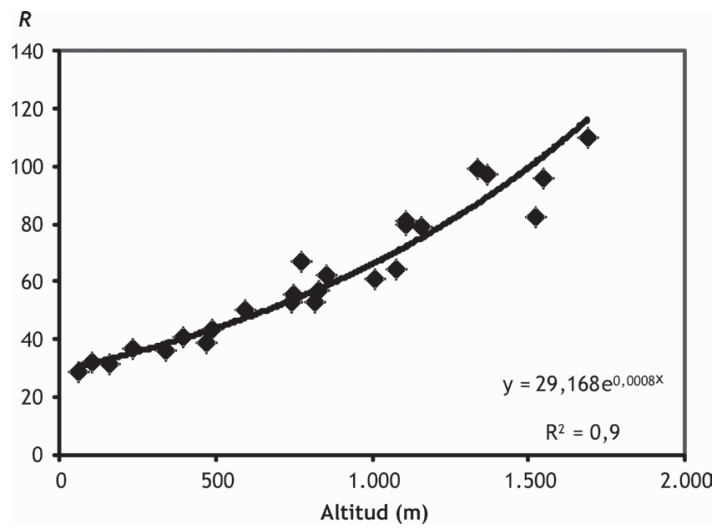

Fig. 2 - Relación entre la altitud y la erosividad de las lluvias (1950-1999).

Fig. 2 - Relationship between altitude and rainfall erosion (1950-1999). 
y permeabilidad y, su cálculo se realiza a partir de la siguiente ecuación:

$100 K=10^{-4} \cdot 2,71 \cdot M^{1,14} \cdot(12-a)+4,20 \cdot(b-2)+3,23 \cdot(c-3)$

Donde $\boldsymbol{M}$ representa el valor de (100 - \% arcilla) (\% limo + arena fina); $\boldsymbol{a}$ es el \% de materia orgánica; $\boldsymbol{b}$ el valor de la estructura del suelo y $c$ representa la clase de permeabilidad del perfil.

Los valores de $\boldsymbol{b}$ se identifican con la siguiente clasificación:

a) Gránulo muy fino y grumo muy fino (<1 mm)

b) Gránulo muy fino y grumo fino $(<1-2 \mathrm{~mm})$

c) Gránulo fino y grumo medio $(2-5 \mathrm{~mm})$ y gránulo grueso $(5-10 \mathrm{~mm})$

d) Gránulo liso, prismático, columnar y muy grueso (> $10 \mathrm{~mm})$

TABLA I - Estaciones pluviométricas, índice de Arnoldus y erosividad calculada ( $R$ ) y estimada ( $\left.R^{\prime}\right)$.

TABLE I - Rainfall stations, Arnoldus index anderoion calculated $(R)$ and estimated ( $R$ ').

\begin{tabular}{|l|c|c|c|c|}
\hline \multicolumn{1}{|c|}{ Estación } & $\begin{array}{c}\text { Altitud } \\
(\mathrm{m})\end{array}$ & $\begin{array}{c}\text { Arnoldus } \\
(\mathrm{Fm})\end{array}$ & $\mathrm{R}$ & $\mathrm{R}$ \\
\hline 3. Las Palmas & 60 & 20,1 & 28,6 & 30,6 \\
\hline 40. Llano de Las Brujas & 100 & 29,4 & 32,0 & 31,6 \\
\hline 38. Lomo del Polvo & 160 & 27,1 & 31,1 & 33,2 \\
\hline 59. San Lorenzo & 235 & 40,5 & 36,5 & 35,2 \\
\hline 66. Tafira Vivero & 337 & 40,2 & 36,4 & 38,2 \\
\hline 102. La Milagrosa & 395 & 50,1 & 41,0 & 40,0 \\
\hline 27. Monte Coello & 470 & 45,1 & 38,6 & 42,5 \\
\hline 29.Santa Brígida & 485 & 55,6 & 43,7 & 43,0 \\
\hline 39. Madroñal & 595 & 67,5 & 50,4 & 46,9 \\
\hline 71. Draguillo-San Mateo & 740 & 71,6 & 52,9 & 52,7 \\
\hline 15. Tenteniguada & 745 & 75,7 & 55,6 & 52,9 \\
\hline 164. Ojero-Teror & 770 & 91,7 & 67,3 & 54,0 \\
\hline 58. Faro Espartero & 818 & 71,7 & 53,0 & 56,1 \\
\hline 136. San Mateo Pueblo & 830 & 77,9 & 57,1 & 56,7 \\
\hline 70. Utiaca & 850 & 85,3 & 62,3 & 57,6 \\
\hline 94. Solana Presa & 1010 & 83,0 & 60,6 & 65,4 \\
\hline 1. Lomo Aljorradero & 1075 & 88,3 & 64,6 & 68,9 \\
\hline 68. Lagunetas & 1110 & 107,2 & 80,9 & 70,9 \\
\hline 69. Antona-Gañanías & 1110 & 106,3 & 80,1 & 70,9 \\
\hline 167. Arínez & 1160 & 105,5 & 79,3 & 73,8 \\
\hline 166. Cueva Grande & 1335 & 124,3 & 99,3 & 84,9 \\
\hline 2. Hoya del Gamonal & 1365 & 122,6 & 97,3 & 86,9 \\
\hline 43. Cruz de Tejeda & 1525 & 108,6 & 82,3 & 98,4 \\
\hline 67. Mesas de Ana López & 1548 & 121,3 & 95,7 & 100,6 \\
\hline 33. Cuevas Blancas & 1690 & 132,9 & 110,0 & 112,7 \\
\hline
\end{tabular}

Los valores de c se clasifican según la siguiente clasificación del USDA-Soil Survey Manual:
a) Rápida a muy rápida
b) Medianamente rápida
c) Moderada
d) Moderadamente lenta
e) Lenta
f) Muy lenta

Los valores de $\mathrm{K}$ para los suelos de la cuenca del Guiniguada se obtuvieron de los realizados por Sánchez et al. (1995), quienes usaron las muestras de suelo tomadas en los siguientes estudios: IRYDA (1981), Sánchez (1975) y Ortega et al. (1991). Esos valores de K se enlazan a la tabla de atributos de las 165 unidades ambientales de la cuenca del Guiniguada, con las que se ha elaborado el mapa de suelos a partir del inventario edafológico de la Cartografía del Potencial del Medio Natural de Gran Canaria (Sánchez et al., 1995). Siguiendo la clasificación de suelos usada en ese trabajo (IUSS Working Group WRB, 2007), en la zona de estudio se identifican 6 de los 32 Grupos de Suelos de Referencia (GSR), que se diferencian según el proceso formador primario que ha producido los rasgos del suelo característicos (TABLA II).

\section{FACTOR LS}

El factor $L S$ expresa cuantitativamente la influencia de la longitud y la pendiente de la ladera en las tasas de erosión del suelo. Se considera que, si no se tienen en cuenta otros aspectos, las pérdidas de suelo por unidad de superficie aumentan al incrementarse la longitud de la ladera, debido a la mayor intensidad que alcanzan determinados procesos con el desarrollo de ésta (mayor velocidad y cantidad de agua de escorrentía). La

TABLA II - Valor de K de los suelos presentes en la cuenca del Guiniguada (adaptado de Sánchez et al., 1995).

TABLE II $-K$ value of the soils present in the Guiniguada (adapted from Sánchez et al., 1995).

Tipo de suelo WRB (FAO). Grupos de Suelos de Referencia (GSR)

\begin{tabular}{|c|c|}
\hline Calcisoles pétricos & 0,45 \\
\hline Leptosoles & 0,42 \\
\hline Calcisoles lúvicos & 0,40 \\
\hline Andosoles vítricos & 0,32 \\
\hline Vertisoles & 0,26 \\
\hline Luvisoles háplicos y ródicos & 0,25 \\
\hline Cambisoles & 0,25 \\
\hline Luvisoles ándicos & 0,19 \\
\hline Andosoles éutricos y dístricos & 0,15 \\
\hline
\end{tabular}


fuente de información utilizada para calcular el factor topográfico $L S$ fue un modelo digital de elevaciones (MDE) disponible en el Centro Nacional de Información Geográfica (CNIG), perteneciente al Instituto Geográfico Nacional de España. La resolución espacial del modelo digital es de $5 \times 5$ metros de celda, y se trabajó con un sistema de referencia de datum WGS84 proyectado en UTM $28 \mathrm{~N}$. El cálculo se realizó en un sistema de información geográfica (SIG), que ofrece la posibilidad, mediante la calculadora ráster, del desarrollo de las fórmulas que se detallan a continuación.

Previamente se elaboran, también a partir del mencionado MDE, los mapas de la red hídrica y de pendiente necesarios para calcular el factor $L S$. Para el primero se usa una función de clasificación de arroyos siguiendo al método de Strahler (1964). El resultado es un mapa en el que aparecen los cursos fluviales identificados según un orden jerárquico, con máximo de orden 6, y el límite topográfico de la cuenca hidrográfica y de las subcuencas. Y para el de pendiente se usan algoritmos implementados en el propio SIG para su cálculo automático. Se identifican ocho intervalos, con amplitudes de clase de $5^{\circ}$, desde las inferiores a los $5^{\circ}$ hasta el umbral superior a $35^{\circ}$.

La formulación original de $L$ se considera como la longitud de la ladera que recorre la gota de escorrentía desde su formación en la divisoria hasta que encuentra un cauce o una zona de sedimentación. Su expresión es:

$$
L=(l / 22,1)^{m}
$$

siendo $L$ el factor de longitud de la ladera, definido como el cociente entre la tasa de erosión anual de una parcela con una longitud $l$ y las tasas de erosión de una parcela con las condiciones de clima, suelo, pendiente, vegetación y prácticas de conservación de una parcela con longitud de ladera igual a 22,1 $m$. El exponente $m$ se corresponde con un valor que depende de la pendiente de la ladera, pudiendo variar entre 0,2 para pendientes inferiores al $1 \%$, y 0,5 para pendientes superiores al $5 \%$.

El factor $S$ expresa la influencia de la pendiente. Su cálculo se deriva de la fórmula:

$$
S=65,41 \cdot \operatorname{sen}^{2} \theta+4,56 \cdot \operatorname{sen} \theta+0,065
$$

donde $S$ es el factor de la pendiente y $\theta$ es el ángulo del declive.

En cuanto al valor topográfico $L S$, a partir de los estudios realizados por McCool et al. (1989), se considera conveniente aumentar la influencia de la longitud de la pendiente $L$, al haberse comprobado su importancia en la formación de regueros. El factor $L$ se calcula a partir de la formulación siguiente:

$$
L=(l / 22,1)^{m}
$$

donde l es la proyección horizontal de la longitud de la ladera y $m$ un factor relativo a la erosión en regueros, que se obtiene de la siguiente manera:

$$
m=B /(1+B)
$$

donde $B$ es el cociente entre la erosión en regueros y la erosión existente entre ellos, que puede evaluarse en función de la pendiente del terreno:

$$
B=(\operatorname{sen} \theta / 0,0896) /\left(3,0 \cdot(\operatorname{sen} \theta)^{0,8}+0,56\right)
$$

siendo $\theta$ el ángulo de la pendiente.

Por su parte, el factor $S$ es hallado según el grado de la pendiente. Si ésta resulta ser mayor al $9 \%$, entonces se aplicará la siguiente fórmula:

$$
S=10,8 \cdot \operatorname{sen} \theta+0,03
$$

Si la pendiente es inferior al 9\%, la fórmula utilizada es:

$$
S=16,8 \cdot \operatorname{sen} \theta-0,50
$$

En las zonas donde el drenaje no origina regueros, la fórmula a utilizar será:

$$
S=3,0 \cdot(\operatorname{sen} \theta)^{0,8}+0,56
$$

Una vez realizados los cálculos, y con el objeto de realizar el mapa correspondiente al factor $L S$, se agrupan los valores obtenidos en 5 categorías o clases: < 2, 2 - 5, $5-10,10-15 y>15$.

\section{FACTOR C}

El factor $C$ representa la influencia de la cobertura vegetal en su acción protectora frente a la fuerza erosiva de las precipitaciones (splash y escorrentía superficial). Se define como el cociente entre la tasa de erosión de una parcela con una determinada cubierta vegetal y la que tendría una parcela con las mismas características de $R, S$ y $L S$, pero en barbecho continuo y labrada según las líneas de máxima pendiente, donde ese valor equivale, al igual que el factor $\boldsymbol{P}$, a la unidad.

En el caso de zonas cultivadas, el valor que se le asigne debe tener en cuenta las prácticas agronómicas del lugar, dado que la función protectora de la vegetación varía a lo largo del año, en función del ciclo vegetativo de las plantas cultivadas y de las labores agrícolas. Por ejemplo, la cobertura del suelo varía estacionalmente si las plantas pierden su hoja, o si el ciclo vegetativo es muy corto, o si el agricultor planta diferentes cultivos durante el año. Por su parte, en ambientes forestales, 
zonas de pasto o áreas de matorral adquieren gran importancia otros aspectos, tales como la altura de la copa o las variaciones fenológicas en la producción de hojarasca u otro tipo de residuos, entre otros.

Los valores de $C$ se han tomado de las tablas resultantes de la adaptación realizada para la isla de Gran Canaria por Sánchez et al. (1995). Éstos tienen en cuenta las diferencias que existen, según los geoambientes, en el tipo de vegetación natural y cultivada, el porcentaje de cobertura sobre el suelo, y el porcentaje de ocupación con respecto a la unidad ambiental.

Las unidades de coberturas del suelo se determinaron a partir de la fotointerpretación y del trabajo de campo. Las fotografías aéreas utilizadas fueron las correspondientes al vuelo de 1961-62, en blanco y negro, a escala 1:16.000 (Cabildo de Gran Canaria), y las ortofotos de 2012 correspondientes al Plan Nacional de Ortofotografía Aérea (PNOA), disponibles en el Centro Nacional de Información Geográfica (CNIG). Esta fuente de información presenta una resolución espacial de 0,25 metros. Los resultados se contrastaron con el mapa de vegetación actual y con el de cultivos, ambos editados por el Gobierno de Canarias. El primero se publica en 2006, con datos de campo obtenidos entre 1999 y 2001, y el segundo con los datos correspondientes a la campaña agrícola 2005.

Para favorecer la buena interpretación visual de los mapas resultantes $\mathrm{y}$, teniendo en cuenta la escala seleccionada (1:50.000), se escoge un tamaño de pixel de $0,25 \times 0,25$ metros.

Los mapas de coberturas del suelo contienen 7 categorías; tres de ellas son agrícolas e indican diferente grado de explotación (en uso, parcialmente en uso y abandonado) y las cuatro restantes, son áreas de pastizal, áreas de matorral, áreas de repoblación y edificación concentrada. Las coberturas con cultivos parcialmente en uso son aquellas en cuyas superficies existe aproximadamente la mitad de campos cultivados y la otra mitad de cultivos abandonados; mientras que los cultivos abandonados abarcan aquellas áreas en las que el abandono agrícola afecta a algo más del $75 \%$ de su superficie.

De la adaptación del valor de $C$ a nuestra área de estudio resultan los valores presentes en la TABLA III.

\section{FACTOR P}

El factor $P$ señala la influencia que tienen las prácticas de conservación de suelos sobre la erosión de las vertientes. Por lo tanto, evalúa la eficacia en el control de la erosión de distintas medidas de conservación tales como terrazas y cultivos a nivel, consideradas como prácticas que cortan las líneas de escorrentía. Se trata de una variable dicotómica, en la que a la presencia de las mismas se le asigna valor 0,5 y a su ausencia, valor 1 .
TABLA III - Valor del factor C en la cuenca del Guiniguada (adaptado de Sánchez et al., 1995).

TABLE III - C factor value in the Guiniguada basin (adapted from Sánchez et al., 1995).

\begin{tabular}{|l|c|}
\hline \multicolumn{2}{|c|}{ Ambiente desértico costero del Norte } \\
\hline TIPO DE VEGETACIÓN & $C$ \\
\hline Tabaibal-cardonal & 0,019 \\
\hline Vegetación degradada & 0,021 \\
\hline Pastizal árido & 0,022 \\
\hline \multicolumn{2}{|c|}{ Ambiente húmedo de medianías } \\
\hline TIPO DE VEGETACIÓN & $C$ \\
\hline Pinar subhúmedo & 0,014 \\
\hline Pinar seco & 0,019 \\
\hline Retamar-jaral & 0,013 \\
\hline Laurisilva & 0,009 \\
\hline Fayal-brezal & 0,009 \\
\hline Brezal & 0,012 \\
\hline Matorral degradado & 0,015 \\
\hline Pastos & 0,015 \\
\hline Eucaliptal & 0,021 \\
\hline & 0,013 \\
\hline Retamar-jaral & 0,013 \\
\hline Escobonal-codesar & 0,013 \\
\hline Tomillar & 0,013 \\
\hline Pastizal & 0,019 \\
\hline Pinar de repoblación & 0,000 \\
\hline TIPO DE CULTIVOS & 0,166 \\
\hline Plataneras & 0,242 \\
\hline Frutales & \\
\hline Policultivo de medianías & \\
\hline Semiabandono o parcialmente en uso & \\
\hline Edificación concentrada & Cumbo cumbre \\
\hline
\end{tabular}

\section{Resultados}

Características de la erosividad de la lluvia, erodibilidad del suelo y de la topografía de la ladera en relación con la erosión hídrica superficial ( $R, K$ y LS) en la cuenca del Guiniguada.

El poder erosivo de la lluvia presenta en esta cuenca valores comprendidos entre 28,6 y 110 (MJ/ha/año) . $(\mathrm{mm} / \mathrm{h}) / 10)$ en las estaciones situadas a menor y mayor altitud, la de Las Palmas y la de Cuevas Blancas, que están a 60 y a 1690 metros de altitud, respectivamente. Los valores altos (80 - $100 \mathrm{Julios} / \mathrm{m}^{2} \cdot \mathrm{mm} / 24$ horas) y muy altos (>100 Julios $/ \mathrm{m}^{2} \cdot \mathrm{mm} / 24$ horas) se localizan en las estaciones pluviométricas situadas en medianías altas (por encima de los 1.100 metros de altitud), en las 
cabeceras meridionales de la cuenca y en la cumbre, lo que representa el $21,66 \%$ de la superficie de la cuenca (TABLA IV y fig. 3).

La erodibilidad del suelo está muy condicionada por la litología y por las geoformas. Los valores más bajos de dicho índice ( $\mathrm{K}<0,18 \mathrm{t}$ ha $\mathrm{h} / \mathrm{ha} \mathrm{MT} \mathrm{mm}$ ) se localizan en suelos jóvenes (Andosoles) de las subcabeceras de la cuenca, formados sobre materiales piroclásticos y que tienen un buen drenaje (arenosos). Entre los suelos que presentan mayor susceptibilidad erosiva se encuentran los Calcisoles y los Vertisoles situados en la costa, con valores de $\mathrm{K}$ comprendidos entre 0,31 y 0,38 , y ocupan el $11,03 \%$ de la superficie de la cuenca (TABLA IV). Los primeros, originados sobre la Formación Detrítica de Las Palmas en condiciones desérticas, presentan altos contenidos en material calcáreo, y se localizan en pendientes acusadas. Por su parte, los Vertisoles aparecen en fondo de barranco, formados sobre materiales aluviales, y muestran altos contenidos en arcillas expansivas.

Por último, casi el $75 \%$ de la superficie de la cuenca presenta suelos con la máxima erodibilidad $(K>0,38)$. Son los Luvisoles de medianías bajas, y los Leptosoles y Cambisoles de medianías altas. De ellos, los Leptosoles son suelos delgados y pedregosos, propios de vertientes con pendientes muy acusadas, y son los más susceptibles a la erosión hídrica. En contrapartida, los Cambisoles y Luvisoles son los suelos con mejores aptitudes agronómicas de toda la cuenca.

Casi la mitad de la superficie de esta cuenca presenta valores moderados $(5$ - 10) del factor topográfico (LS), exactamente el 49,19\% de su superficie (TABLA IV). Los valores más elevados se localizan en las vertientes de los barrancos más antiguos, donde el grado de encajamiento es mayor y en las cabeceras de la cuenca, donde las pendientes son más acusadas $\left(>20^{\circ}\right)$ debido al gradiente altimétrico que deben salvar en esos sectores, y representan el 19,03\% de superficie de la cuenca.
Características de los factores socioeconómicos de la erosión hídrica superficial ( $P$ y $C$ ) en la cuenca del Guiniguada.

La práctica de conservación de suelos más extendida en la cuenca del Guiniguada es el escalonamiento de sus vertientes con terrazas de cultivo (fig. 4). Casi la mitad de la superficie de esta cuenca está ocupada por terrazas agrícolas o bancales $(46,5 \%)$ que representan, a su vez, el $81,6 \%$ de la superficie agrícola. Estos datos ponen de manifiesto el valioso patrimonio en terrazas agrícolas de la cuenca del Guiniguada que alertan sobre la vulnerabilidad de sus vertientes frente a la erosión. Las terrazas agrícolas se localizan sobre las vertientes de todos los geoambientes de esta cuenca, por lo que participan de la variedad de condiciones ambientales que en ella existen (Romero et al., 2007a y 2007b). Es en las medianías donde alcanzan su máxima extensión, con una leve diferencia a favor de las medianías bajas respecto de las altas (fig. 5).

Los mapas de coberturas del suelo realizados aportan información acerca del grado de intervención humana sobre el territorio en 1960 y 2012 y de la protección del suelo por la vegetación (natural y cultivada). En 1960, el $61 \%$ de la superficie de la cuenca estaba ocupada por cultivos (fig. 6 y 7) y el 35\% por coberturas vegetales naturales. La cobertura antrópica dominante era la de cultivos en uso (50\%), mientras que la de edificación concentrada suponía tan sólo el $3 \%$ de la superficie. Los cultivos abandonados y los parcialmente en uso representaban sólo el $8 \%$ y el $3 \%$ de la superficie de la cuenca. Estos datos indican que los procesos de abandono y de extensificación agrícola eran incipientes. Entre las coberturas naturales destacaba el matorral que se extendía por toda la cuenca y tenía, como rasgo común, su escasa densidad, por lo que grado de protección del suelo debió ser reducido.

La distribución de las coberturas del suelo, según los geoambientes, muestra que en 1960 era máxima la extensión de los cultivos en uso en medianías bajas y costa (más de la mitad de sus superficies), y mínima en

TABLA IV - Distribución superficial (\%) de los factores R, K y LS en la cuenca del Guiniguada.

$T_{A B L E} I V$ - Surface distribution (\%) of the $R, K$ and LS in the Guiniguada basin.

\begin{tabular}{|c|c|c|c|c|c|}
\hline Factor $R$ & Superficie (\%) & Factor $K$ & Superficie $\%$ & Factor $L S$ & Superficie $\%$ \\
\hline$<40$ & 22,80 & $<0,18$ & 4,68 & $<2$ & 19,35 \\
$40-60$ & 36,51 & $0,18-0,25$ & 8,42 & $2-5$ & 20,43 \\
$60-80$ & 19,02 & $0,25-0,31$ & 1,35 & $5-10$ & 41,19 \\
$80-100$ & 13,96 & $0,31-0,38$ & 11,03 & $10-15$ & 16,70 \\
$>100$ & 7,70 & $0,38-0,45$ & 49,49 & $>15$ & 2,33 \\
& & $>0,45$ & 25,03 & & \\
\hline Total & 100,00 & Total & 100,00 & Total & 100,00 \\
\hline
\end{tabular}



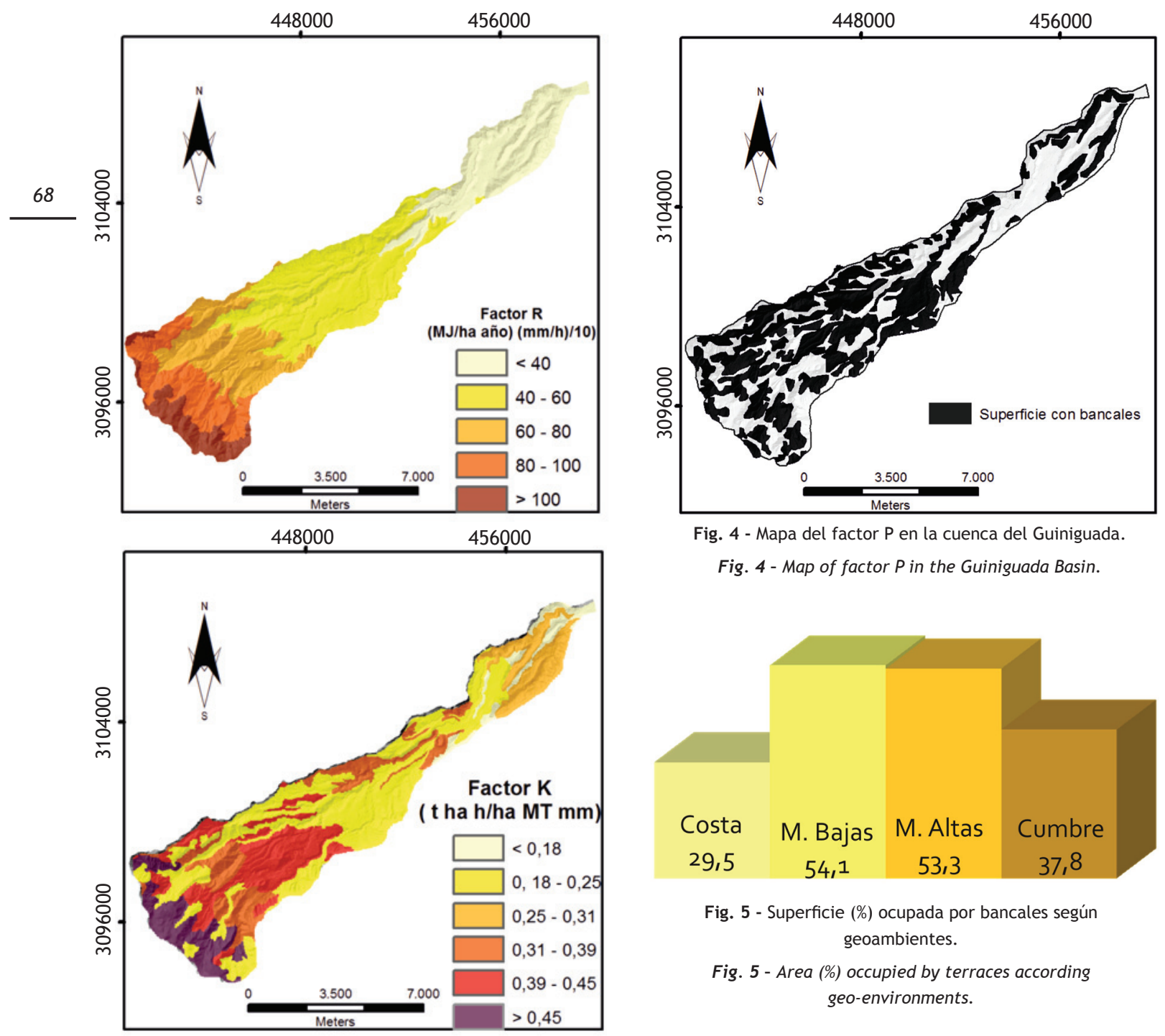

Fig. 4 - Mapa del factor $P$ en la cuenca del Guiniguada.

Fig. 4 - Map of factor $P$ in the Guiniguada Basin.

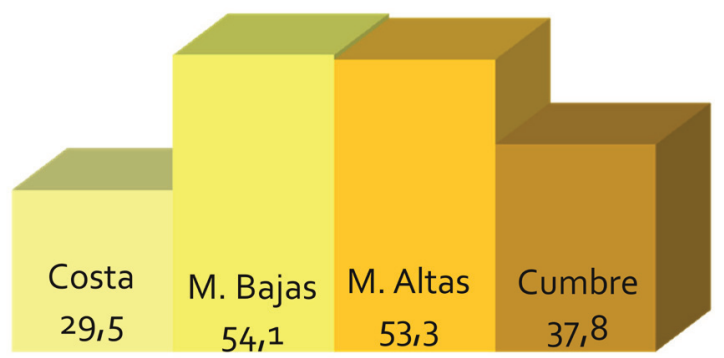

Fig. 5 - Superficie (\%) ocupada por bancales según geoambientes.

Fig. 5 - Area (\%) occupied by terraces according geo-environments.
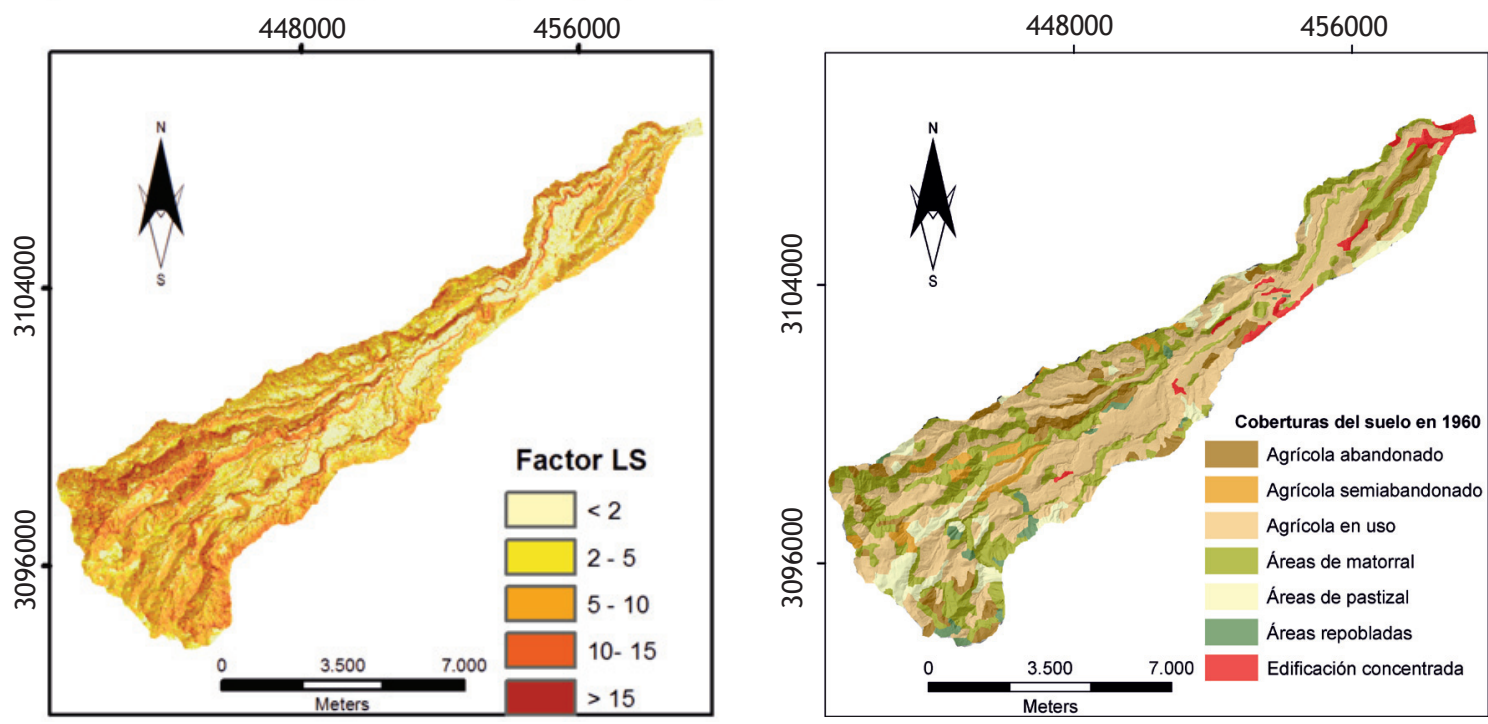

Fig. 3 - Mapas de factores físicos de la USLE en la cuenca del Guiniguada.

Fig. 6 - Mapa de coberturas del suelo. Cuenca del Guiniguada (1960).

Fig. 6 - Map of land cover. Guiniguada Basin (1960). 


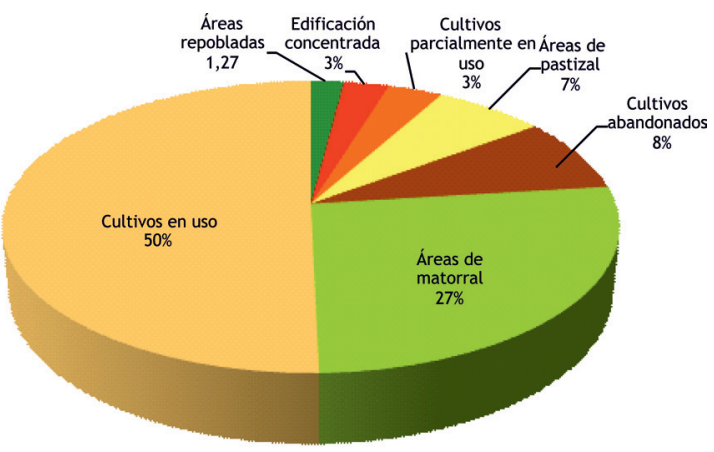

Fig. 7 - Surface distribution of land cover (\%). Guiniguada Basin (1960).

Fig. 7 - Distribución superficial de las coberturas del suelo (\%). Guiniguada Basin (1960).

la cumbre (26\% de su superficie) (fig.8). Por su parte, los espacios agrícolas abandonados se correspondían con áreas marginales, de escasa productividad agrícola por la calidad de sus suelos, o por ser de difícil accesibilidad, localizándose en las áreas de costa y de cumbre. Finalmente, las áreas repobladas y de pastizal alcanzaban su máxima extensión en la cumbre.

El año 1960 representa el momento de máxima extensión y dedicación agrícola de la cuenca del Guiniguada, por lo que los valores del factor $C$, en ese momento, están condicionados, por una parte, por el tipo de cultivo que ocupa una superficie mayor, los herbáceos; y, por otra, por la vegetación natural, en la que predomina el matorral abierto, con menor capacidad para controlar la erosión hídrica.

En 2012 las coberturas agrícolas suponían el 49\% de la superficie de la cuenca, las de la vegetación natural, el $40 \%$, y la edificación concentrada, el $11 \%$ restante (fig. 9 y 10). Entre las coberturas agrícolas, las superficies abandonadas se extendían por el $15 \%$ de la cuenca, y los cultivos parcialmente en uso, por el $14 \%$ de la misma. Estos últimos valores evidencian la pérdida de peso económico de la actividad agrícola en este espacio. Por otro lado, el incremento de las coberturas de matorral (34\%) y de la vegetación arbórea (5\%) es consecuencia de la recolonización natural de espacios agrícolas abandonados en unos casos, y de las políticas de repoblación practicadas por la administración, en otros.

La distribución de las coberturas por geoambientes en 2012 pone de manifiesto grandes diferencias entre ellos (fig. 11). El geombiente que mantiene un mayor porcentaje de cultivos en uso es el de las medianías altas (28,7\% de su superficie); de cultivos parcialmente en uso es el de las medianías bajas (30,1\%); y de abandono, los ambientes de costa y medianías bajas. La edificación concentrada alcanza en 2012 su máxima extensión en la costa $(30,1 \%$ de su superficie) y en medianías bajas

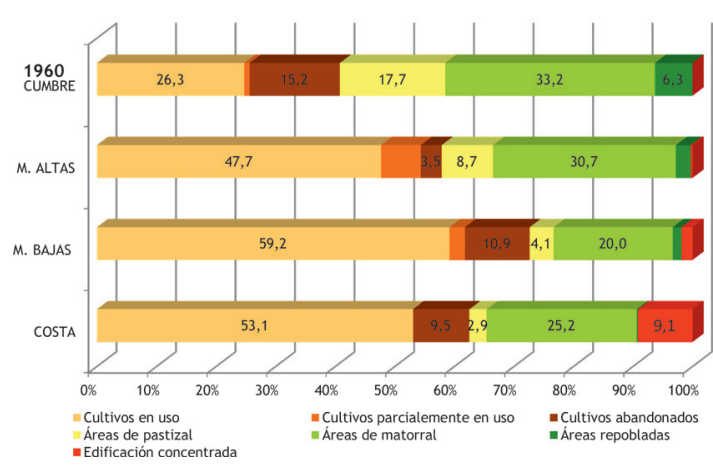

Fig. 8 - Distribución superficial de las coberturas del suelo (\%) por geoambientes (1960).

Fig. 8 - Surface distribution of land cover (\%) by geo-environments (1960).

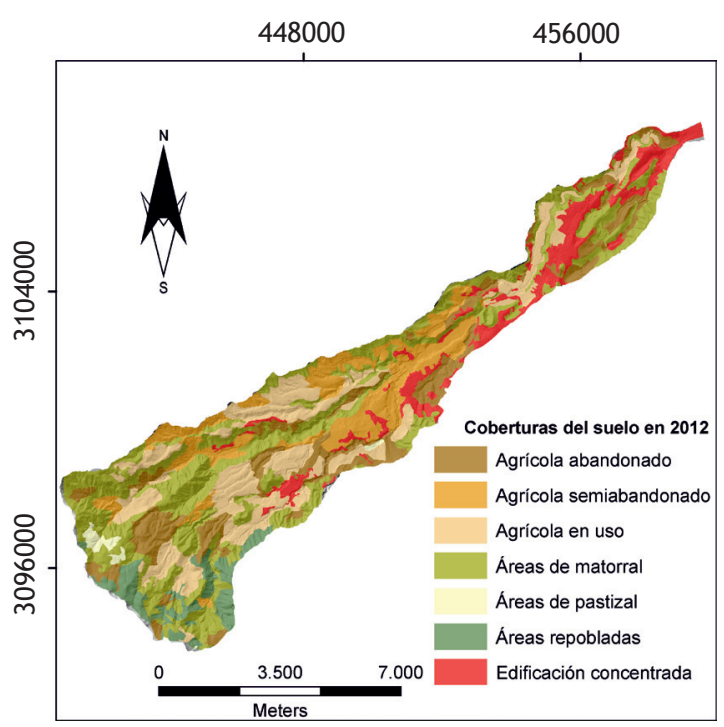

Fig. 9 - Mapa de coberturas del suelo. Cuenca del Guiniguada (2012).

Fig. 9 - Map of land cover. Guiniguada Basin (2012).

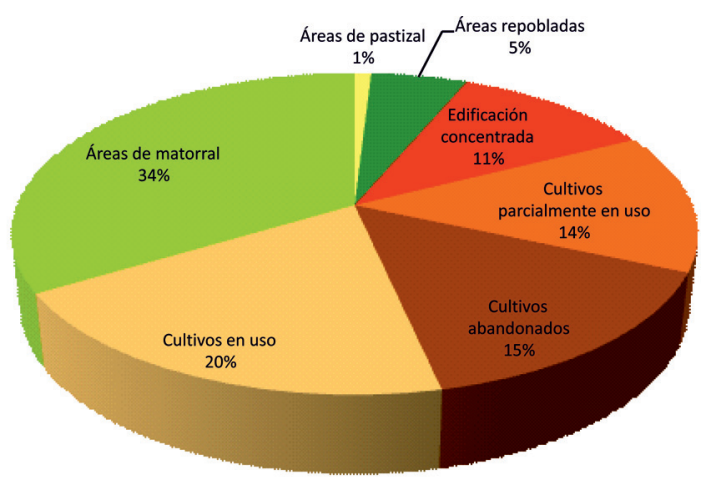

Fig. 10 - Distribución superficial de las coberturas del suelo (\%). Cuenca del Guiniguada (2012).

Fig. 10 - Surface distribution of land cover (\%). Guiniguada Basin (2012). 


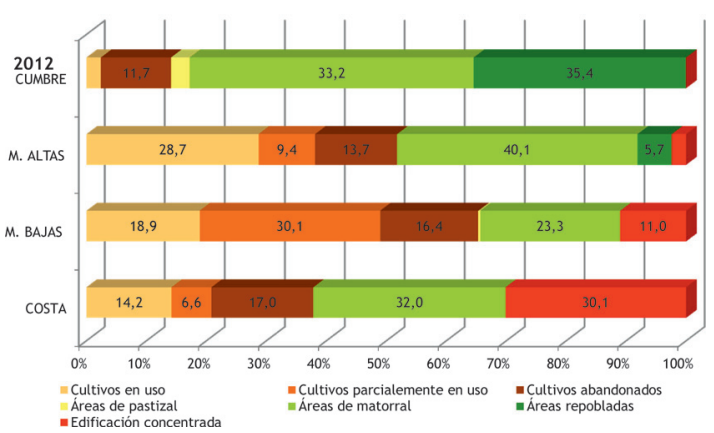

Fig. 11 - Distribución superficial de las coberturas del suelo (\%) por geoambientes (2012).

Fig. 11 - Surface distribution of land cover (\%) by geo-environments (2012).

(11\%), debido a la expansión del área metropolitana de Las Palmas de Gran Canaria. Finalmente, las coberturas de matorral y de repoblación presentan su máxima extensión en los geoambientes del interior de la cuenca, en las medianías altas y en la cumbre.

Los procesos de cambio en esta etapa son, fundamentalmente, el abandono agrícola, la erialización y matorralización de antiguas superficies de cultivo, la extensificación agrícola $y$, por último, dos procesos antagónicos: la artificialización, especialmente por la edificación y los nuevos viarios y, por otro la repoblación de extensas zonas de la cabecera de la cuenca.

El año 2012 representa el momento en el que la ocupación agrícola de la cuenca es mínima y, en contrapartida, es máxima la de la vegetación natural (arbustiva fundamentalmente) y la de la edificación concentrada. Los valores del factor $C$ en ese momento están condicionados por el tipo de vegetación que se desarrolla en cada geoambiente, con mayor capacidad para proteger ante la erosión en las medianías altas y en la cumbre.

Los cambios experimentados por las coberturas del suelo, entre 1960 y 2012, ponen de manifiesto la desagrarización de esta cuenca, junto a la artificialización, en unos sectores, y la naturalización en otros (fig.12). De lo primero da cuenta la reducción, a más de la mitad, de la superficie de cultivos en uso, que pasan del 49,6\% (1960) al 19,8\% (2012), así como el incremento de las superficies con cultivos parcialmente en uso y con cultivos abandonados. Por su parte, los procesos de reforestación y los de artificialización justifican el incremento de la superficie ocupada por las coberturas relacionadas con ellos (áreas repobladas y áreas edificadas), que se duplican o triplican en el área analizada durante los 52 años estudiados.

Una vez constatados los cambios de cobertura, se calculan las tasas de erosión para cada año y se realiza el balance de las pérdidas de suelo entre 1960 y 2012.

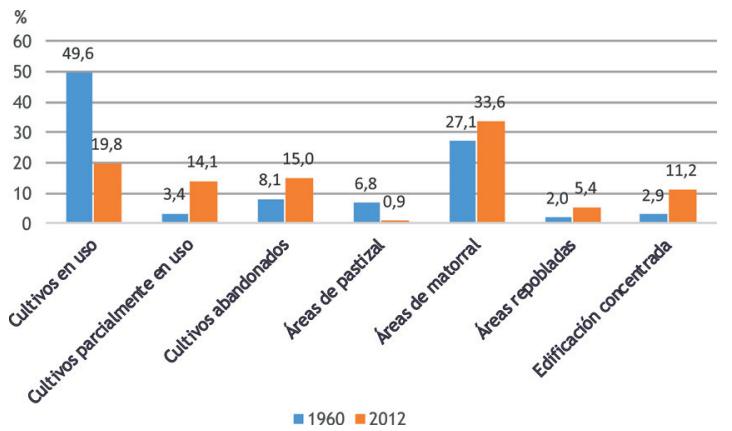

Fig. 12 - Evolución de las coberturas del suelo (1960 - 2012).

Fig. 12 - Evolution of land cover (1960 - 2012).

Con ello se pretende determinar si las mencionadas tasas han variado en el intervalo temporal considerado. En 1960 la erosión media de la cuenca fue de 18,6 t/ha/ año (fig. 13 y TABLA V) y el $52,6 \%$ de la superficie de la cuenca registra tasas bajas y moderadas de pérdidas de suelo, comprendidas entre 10 y 50 t/ha/año. En 2012 la erosión media de la cuenca se reduce levemente al 16,1 t/ha/año, y en el 55,4\% de la superficie de la cuenca se registran tasas de erosión inferiores a 10 t/ha/año. En el balance final se comprueba un incremento notable de las superficies con tasas de erosión muy bajas (>0,1-10 t/ha/ año), e incrementos leves en los umbrales más elevados de las tasas de erosión (>50 t/ha/año) que afectan al 5,2\% de la superficie de la misma en 2012.

El análisis de las pérdidas de erosión por geoambientes (fig. 14) permite matizar lo indicado en el párrafo anterior, pues si bien es cierto que la tasa de erosión media se reduce si se considera toda la cuenca, la tendencia no es la misma en todos los geoambientes. La reducción más significativa de la erosión se produce en la cumbre, cuestión que se debe a la combinación de los efectos de la recolonización espontánea de la vegetación y de la plantación de especies arbóreas en los antiguos campos abandonados. Por su parte, las superficies que en 2012 registran las tasas de erosión más elevadas (>50Tm/ ha/año) se localizan en las medianías altas, afectando al 9,2\% de su superficie frente al 5\% de la misma en el año inicial. Ese incremento significativo de las tasas de erosión altas se debe al abandono agrícola de su amplia superficie abancalada que triplica extensión, desde el $3,51 \%$ en 1960 al $13,7 \%$ en 2012.

El análisis del balance erosivo (incremento o decremento de las pérdidas de suelo) muestra una cuenca en la que en más de la mitad de su superficie (el 65,1\%) han aumentado las tasas de erosión entre 1960 y 2012 (fig. 15). En el 34,9\% restante se ha producido el proceso inverso. Además, se aprecia un balance muy desequilibrado: predominio del incremento en las medianías altas y, en menor medida, en las medianías bajas (fig. 16). En el primer caso, puede deberse al abandono agrícola y, en el segundo, a la extensificación agrícola, pues en 2012 

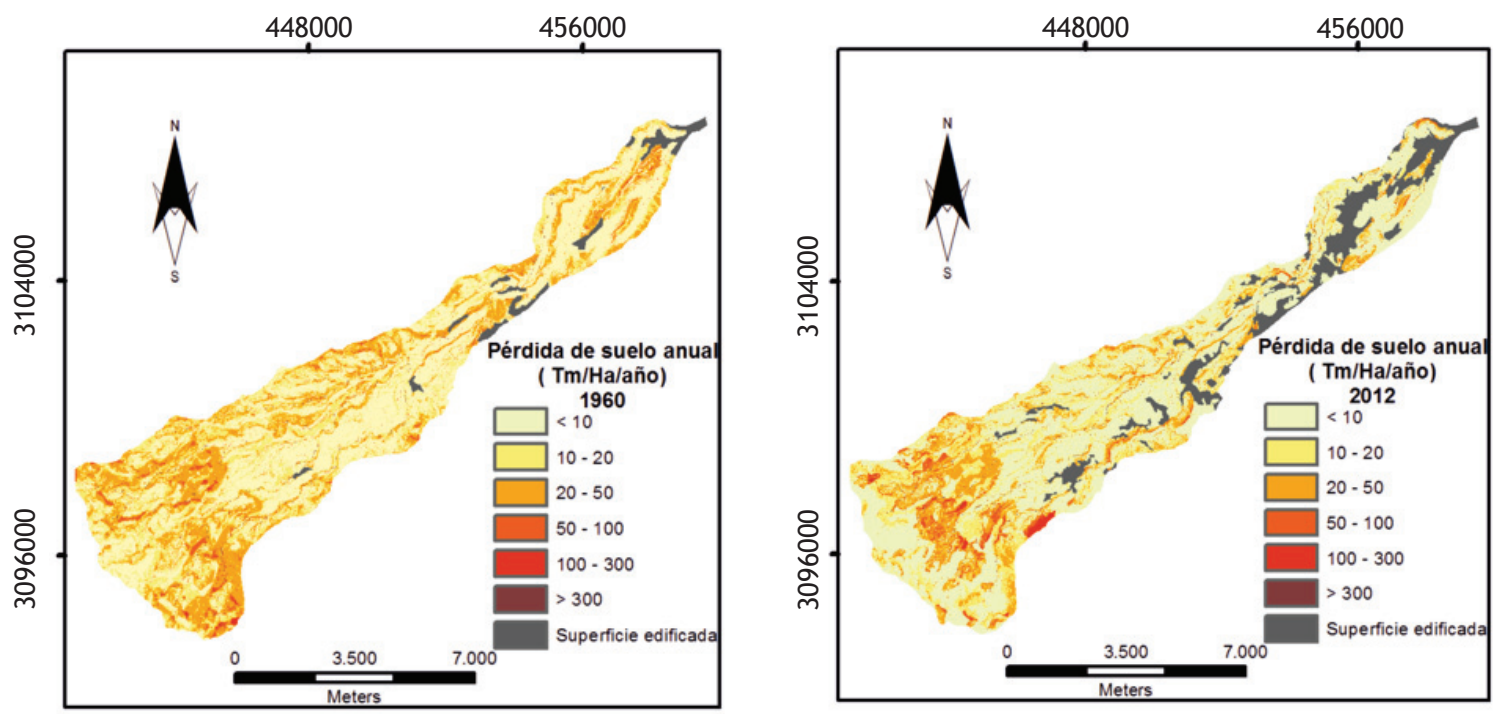

Fig. 13 - Mapas de tasas de erosión (1960 y 2012). Cuenca del Guiniguada

Fig. 13 - Maps of erosion rates (1960 and 2012). Guiniguada Basin.

TABLA V - Evolución de las tasas de erosión (1960 - 2012).

$T_{A B L E} V$ - Evolution of erosion rates (1960 - 2012).

\begin{tabular}{|l|c|c|c|c|}
\hline \multicolumn{1}{|c|}{ Tasas } & t/ha/año & 1960 & 2012 & $1960-2012$ \\
\hline Muy baja & $>0,1-10$ & 43,41 & 55,42 & 12,01 \\
\hline Baja & $>10-20$ & 26,94 & 22,42 & $-4,52$ \\
\hline Media & $>20-50$ & 25,71 & 16,96 & $-8,75$ \\
\hline Alta & $>50-100$ & 3,15 & 3,74 & 0,58 \\
\hline Muy alta & $>100-300$ & 0,54 & 1,21 & 0,67 \\
\hline Irreversible & $>300$ & 0,24 & 0,25 & 0,01 \\
\hline Erosión media de la cuenca t/ha/año & & 18,6 & 16,1 & $-2,5$ \\
\hline
\end{tabular}
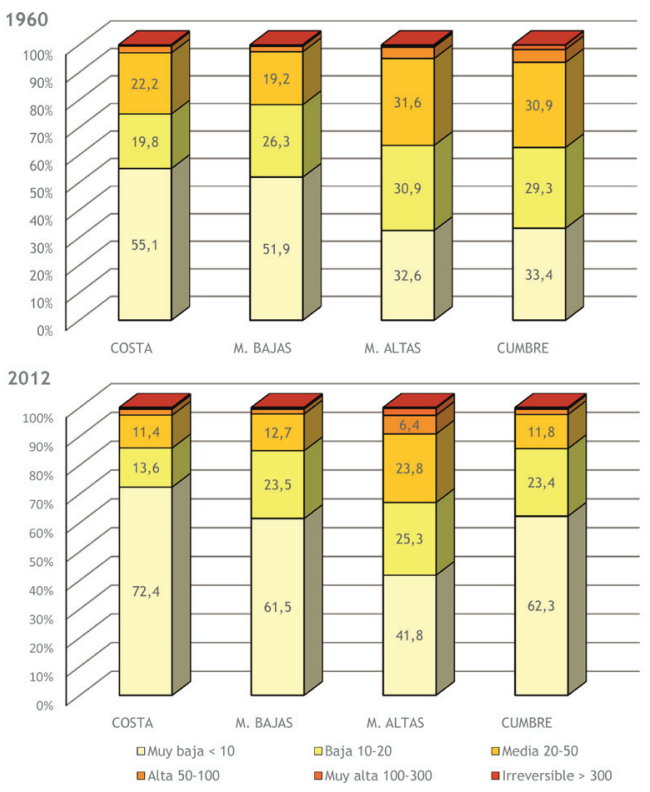

Fig. 14 - Evolución de las tasas de erosión (t/ha/año) por geoambientes (1960 - 2012).

Fig. 14 - Evolution of erosion rates ( $t /$ ha/year) by geo-environments (1960 - 2012).

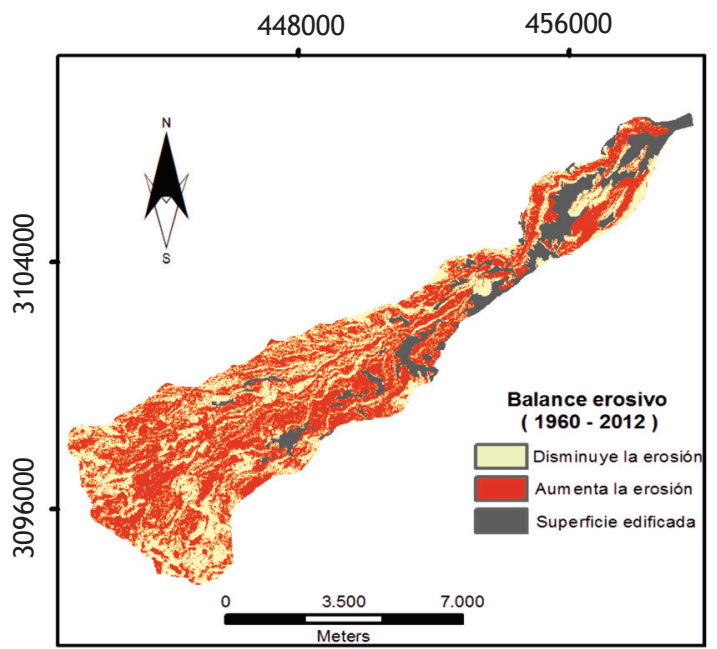

Fig. 15 - Mapa de balance erosivo (1960 - 2012).

Fig. 15 - Map of erosive balance (1960 - 2012).

la superficie de cultivos en uso parcial representaba, en ese geoambiente, el $30,1 \%$ de su extensión frente al 2,6\% de inicios del periodo de estudio. 


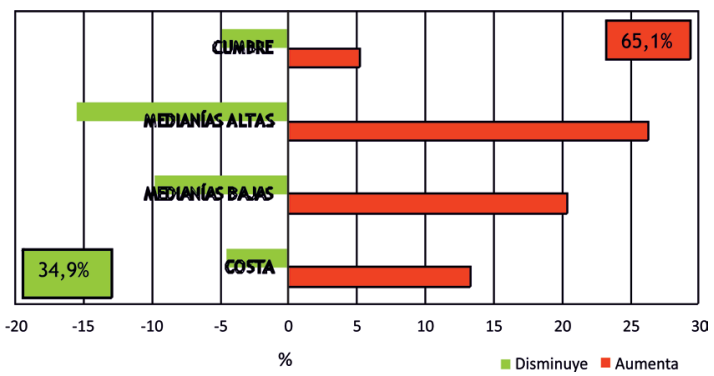

Fig. 16 - Balance erosivo por geoambientes (\%) (1960-2012).

Fig. 16 - Erosive Balance by geo-environments (\%) (1960-2012).

Por otro lado, si se introduce el concepto de erosión tolerable o tasa máxima de erosión permisible para que la fertilidad del suelo pueda mantenerse durante 20 ó 25 años y se admite el valor de $10 \mathrm{t} / \mathrm{ha} / \mathrm{año}$ como umbral de máxima pérdida tolerable (Wischmeier y Smith, 1978 y Geler et al., 2004), se comprueba que ésta se ha incrementado en un $7,1 \%$ mientras que la no tolerable ha descendido en un $15,5 \%$ de la superficie de esta cuenca ( $T_{A B L A}$ VI). Pero esa evolución positiva señalada no debe enmascarar el elevado porcentaje de superficie de esta cuenca, concretamente el 39,9\% que, en 2012 registra tasas de erosión no tolerable y que nos alerta sobre el riesgo de descenso de la productividad agrícola de la misma.

TABLA VI - Evolución de las superficies de erosión tolerable y no tolerable (1960 - 2012). Cuenca del Guiniguada.

$T_{A B L E}$ VI - Evolution of surface no tolerable and tolerable erosion (1960-2012). Guiniguada Basin.

\begin{tabular}{|c|c|c|c|}
\hline Superficie \% & 1960 & 2012 & $1960-2012$ \\
\hline Edificado & 2,7 & 11,1 & 8,3 \\
\hline $\begin{array}{c}\text { Tolerable } \\
(<10 t / \text { ha/año })\end{array}$ & 41,9 & 49,0 & 7,1 \\
\hline $\begin{array}{c}\text { No Tolerable } \\
(>10 t / \text { ha/año })\end{array}$ & 55,4 & 39,9 & $-15,5$ \\
\hline
\end{tabular}

\section{Discusión}

Los cambios de usos del suelo se manifiestan en el territorio de dos maneras diferentes: a corto plazo, transformando las coberturas del suelo $y$, a medio y largo plazo, modificando los balances de agua y sedimentos como respuesta del medio ante esos cambios. En este último sentido, el signo y la magnitud de dichos balances dependen de las características de los cambios que se han producido y de las del propio medio (Lasanta et al., 2000; Sánchez-Bosch y Martínez-Casanovas, 2000 y Seeger y Beguería, 2003).

Los resultados muestran cambios sustanciales en las coberturas del suelo que resultan de la evolución de un modelo económico agrario hacia otro urbanoturístico, iniciado en los años sesenta del siglo XX. Las manifestaciones más importantes de dichos cambios son: la reducción de la superficie cultivada, el incremento de las coberturas naturales (recolonización de matorral y reforestación arbórea) y de las superficies urbanas. Dichos cambios se manifiestan de desigual modo según los geoambientes. La pérdida de superficie agrícola es más evidente en los geoambientes extremos de la cuenca (costa y cumbre). En el primero, debido al notable incremento de la superficie urbana y de viales $y$, en el segundo, por los procesos de naturalización (recolonización de matorral y reforestación). Las medianías son los geoambientes que, al final del periodo de análisis, conservan la mayor superficie agrícola de la cuenca, pero con desigual grado de explotación: en las medianías altas se mantiene en cultivo el $28,7 \%$ de su superficie, y en las medianías bajas tienen el 30,15\% de la misma con cultivos parcialmente en uso.

Los cambios señalados repercuten en las tasas de erosión registradas en ambos años y en la evolución de las mismas a lo largo del periodo de análisis. En 1960 el $52,6 \%$ de la superficie de la cuenca presenta pérdidas de suelo bajas-medias (10-50 t/ha/año); en 2012, en el $55,42 \%$ de la misma, las tasas erosivas responden a la categoría de muy baja (>0,1-10 t/ha/año) debido al notable incremento de la cobertura de matorral y de las superficies reforestadas. La erosión media de la cuenca en 1960 es de 18,62 t/ha/año y en 2012 de 16,10 t/ha/año. Estos resultados son similares a los obtenidos por otros autores (Hernández-Rodríguez, et al., 2004) que aplican el mismo método en otros ámbitos geográficos, pues señalan una reducción de la erosión media del 17,64\% en la parte media y alta de la cuenca del río Corneja (Ávila), que pasa de 34,80 t/ha/año en 1957 a de 28,66 t/ha/año en 2013. Dicha reducción es consecuencia de la restauración hidrológico-forestal llevada a cabo desde 1964, por la Confederación Hidrógrafica del Duero. Esta práctica ha transformado el paisaje, favorecido el desarrollo de una cubierta vegetal que ha reducido la escorrentía superficial y los caudales punta de dicho río.

En el caso de la cuenca del Guiniguada, los valores erosivos del primer intervalo temporal considerado, el de máxima dedicación agrícola de la cuenca están relacionados con la baja capacidad de los cultivos dominantes en la cuenca (hortalizas y papas) para proteger el suelo. Éstos cultivos tienen un ciclo vegetativo corto, por lo que dejan al descubierto el suelo durante varios meses al año, al tiempo que su sistema de enraizamiento retiene poco el suelo. Por su parte, los valores obtenidos en 2012, se explican por el incremento de la densidad y extensión superficial de las coberturas vegetales naturales (matorral y arbórea) en toda la cuenca. No obstante, es necesario matizar este aspecto, pues el grado de protección varía según el geoambiente del que se trate, siendo máximo en la cumbre. Ello se explica porque ahí la recolonización vegetal presenta facies muy evolucionadas (arbustivas y arbóreas), a ser 
más antiguo el abandono de los cultivos. A ello se suma el hecho de que amplias zonas han sido repobladas con vegetación arbórea.

Los datos de la evolución de las tasas de erosión permiten considerar que el proceso de abandono agrario no ha perjudicado a la cuenca hidrográfica en su conjunto, pues los incrementos señalados entran dentro de los límites considerados por algunos autores como "erosión tolerable". Se entiende como tal a la cantidad de tierra que puede perder un perfil edáfico manteniendo su nivel de productividad actual durante un largo periodo de años (F.A.O., 1967). Este concepto indica la máxima pérdida de suelo admisible para que tenga un grado de conservación tal que permita una producción económica similar, con los medios técnicos disponibles en la actualidad. Según los autores señalados, se consideran tasas de erosión tolerables, las que se encuentran entre 5 y 10 t/ha/año.

Tomando ese umbral de referencia y, sin llegar a considerar si es o no adecuado su empleo en nuestra área de estudio (isla de reducidas dimensiones, densamente poblada, con escasos suelos agrícolas y alta dependencia alimentaria del exterior), se comprueba que en la cuenca se produce, durante el periodo estudiado, una reducción notable de la superficies con tasas de erosión "no tolerable" (del 15,5\%) aunque esa categoría se mantiene todavía en un $39,9 \%$ de la superficie de la cuenca del Guiniguada.

Desde el punto de vista metodológico, el procedimiento empleado (USLE), junto a su implementación mediante el uso de los SIG, ha resultado de gran utilidad para la consecución de los objetivos propuestos, pues ha permitido conocer la evolución y el balance de las tasas de erosión en el período de análisis, su distribución espacial, así como identificar las áreas de máxima vulnerabilidad erosiva actual de la cuenca. En este sentido destacan las medianías altas, pues es el geoambiente que registra la proporción mayor de superficie con pérdidas erosivas altas en 2012: el 32,9\% del geoambiente registra pérdidas de suelo superiores a las $20 \mathrm{t} / \mathrm{ha} / \mathrm{año}$. Conviene destacar que precisamente ese ámbito es el que presenta mayor agrodiversidad y superficie abancalada de toda la cuenca del Guiniguada.

Por último, procede hacer una serie de reflexiones con respecto a la consideración que reciben, en este método, los factores $(C$ y $P$ ) utilizados para el cálculo de la erosión en la cuenca objeto de estudio. Con respecto al factor $C$, cabe señalar que resultó complejo su cálculo, debido a la gran heterogeneidad de coberturas y ocupaciones del suelo por unidad de superficie. Además, se trata de un territorio con amplias extensiones de superficies agrícolas abandonadas que, según la edad de abandono, el modelo de campo o las condiciones ambientales (clima, suelos, topografía), presentan diferentes etapas de recolonización vegetal. Este paisaje en mosaico complica la determinación exacta del factor C. Sería adecuado contemplar escalas de mucho mayor detalle a las empleadas en este trabajo. La teledetección ha sido una herramienta sumamente eficaz en otros territorios, pero no parece serlo tanto en el estudiado, pues se trata de un espacio muy abrupto, donde las teselas de cobertura de suelo son muy pequeñas, por lo que sería necesario contar con imágenes de satélite de alta resolución cuyo coste es elevado.

Por otra parte, y por lo que respecta al factor $P$ (prácticas de conservación de suelos) se ha constatado que en la zona de estudio, dada su diversidad de tipos de campo, no es suficiente valorar este factor en términos de presencia/ausencia, como se plantea en la USLE. La eficacia de los bancales ante la erosión varía según los modelos de estructuras abancaladas (pendiente del rellano, tipo de muro o talud, anchura del rellano, altura del muro) y el grado de ocupación en la ladera, entre otros. Por ello se considera que sería importante incluir, en un modelo erosivo como el planteado, algunas de estas características, con la finalidad de que sus resultados se ajusten más a la realidad.

En ese sentido $y$, siguiendo las apreciaciones de Vaudour y Bonin (1991), quienes señalan que la degradación de las laderas aterrazadas siguen dos ritmos evolutivos, se aprecia que la extensa superficie abancalada de la cuenca del Guiniguada ha seguido, en los 52 años que comprende este estudio, un ritmo lento de degradación, gracias a la funcionalidad e inercia de estas estructuras antierosivas. Pero ese lento ritmo puede acelerarse en el momento en que se produzca la instalación de los procesos erosivos que más contribuyen a su degradación, los movimientos en masa de sus muros (García-Ruiz y López-Bermúdez, 2009). Es en ese momento cuando se reduce, con un ritmo acelerado, la eficacia de estas medidas que permiten la conservación del suelo.

\section{Conclusiones}

En la cuenca del Guiniguada, tras el proceso de abandono agrario experimentado, se constata que las pérdidas de suelo consideradas "no tolerables" han descendido en las últimas cinco décadas. Ello se debe al proceso de naturalización (matorralización y repoblación) que sucede al abandono agrario. No obstante, el 39,9\% de la superficie de la cuenca todavía registra valores de erosión "no tolerable”. Las áreas que muestran en 2012 una mayor vulnerabilidd erosiva son las medianías altas, en las que se encuentran los mejores suelos agrícolas y la máxima superficie abancalada de toda la cuenca. Todo ello pone en riesgo la conservación de un paisaje singular (el paisaje agrícola en bancales), así como la calidad y la productividad de los escasos suelos agrícolas que existen. 
Al mismo tiempo se aleja el objetivo de conseguir la soberanía alimentaria de un territorio insular, distante de los centros de provisión de alimentos.

\section{Bibliografía}

Abadín, Y., Pejenaute, I., Romero, L. E., Ruiz-Flaño, P., Pérez-Chacón, E. y Camarasa, A. (2004). Análisis de la evolución de las tasas de erosión de suelos mediante SIG. Aplicación a la cuenca del barranco de Guiniguada, Gran Canaria, Islas Canarias (1960-2002). En: Conesa, C. y Martínez, J. B. (Eds.) Territorio y Medio Ambiente: Métodos Cuantitativos y Técnicas de Información Geográfica, p. 127-138.

Abadín, Y. (2007). Aplicación de un Sistema de Información Geográfica (SIG) al análisis evolutivo de la erosión de suelos como consecuencia de los cambios de uso en la isla de Gran Canaria. Vector Plus, 29, p. 68-77.

Agnoletti, M. (2006). The development of a historical and cultural evaluation approach in landscape assesment: the dynamic of Tuscan landscape between 1832 and 2004. En: Agnoletti, A. (Ed.) The Conservation of Cultural Landscapes, p. 3-41.

Agnoletti, M. (2007). The degradation of traditional landscape in a mountain area of Tuscany during the 19th and 20th centuries: Implications for biodiversity and sustainable management. Forest Ecology and Management, 249, p. 5-17.

Araneda, A. (2002). Cuantificación de la erosión histórica, mediante SIG y USLE, en una Cuenca lacustre sometida a cambios de uso del suelo. Universidad de Concepción. Chile. Consultado 4 nov. 2002. Disponible en: http//www.ucv.cl./web/geografía/com4.htm.

Arévalo, D., Bienes, R. y Ruiz, M. (2011). Aplicación de modelos de erosión en el centro de la Península Ibérica: incorporación y evaluación de nuevos parámetros con influencia en las pérdidas de suelo. IV Congreso Ibérico de la Ciencia del Suelo. Granada, p. 552-564.

De Antonio, R. (1994). Evaluación de la erosión hídrica mediante sistemas de información geográfica. Aplicación a la Comunidad de Madrid (Tesis Doctoral). E.T.S.L.A. Agrónomos. Madrid, España. Universidad Politécnica de Madrid, 188 p.

Delgado Sánchez, J. C., del Palacio Fernández-Montes, E. (2005). El Inventario Nacional de Erosión de Suelos: perspectiva general, situación actual, previsiones de futuro y principales resultados e indicadores obtenidos. Actas IV Congreso Forestal Español, Zaragoza, 26 - 30 septiembre. Ed. Sociedad Española de Ciencias Forestales. Disponible en: www.congresoforestal.es.
Dumas, A. (2012). Riesgo de erosión hídrica en la cuenca hidrográfica del rio Mundo (Trabajo de Fin de Master). Master en Tecnologías de la Información Geográfica. Universidad Complutense de Madrid. Madrid, 48 p.

Erdogan, E. H, Erpul, G. y Bayramin, I. (2007). Use of USLE/GIS methodology for predicting soil loss in a semi-arid agricultural watershed. Environ. Monit. Assess., 131, pp: 153-161.

Errea, M. P., Lasanta, T., Arnáez, J., Ortigosa, L., RuizFlaño, P. y Oserín, M. (2009). Cambios en el paisaje de Cameros durante la segunda mitad del siglo XX. En: Lasanta, T. y Arnáez, J. (Eds.) Gestión, usos del suelo y paisaje en Cameros (Sistema Ibérico, La Rioja), p. 165-190. Logroño. Universidad de La Rioja e Instituto de Estudios Riojanos.

F.A.O. (1967). La Erosión del Suelo por el Agua: Algunas medidas para combatirlas en las tierras de cultivo. Colección F.A.O: Fomento de Tierras y Aguas, n7. FAO, Roma (También FAO: Cuadernos de fomento agropecuario $n^{\circ} 81$ )

Fernández García, F. (1995). Manual de climatología aplicada: clima, medio ambiente y planificación. Síntesis.

Fernández-Palacios, J. M., Arévalo, J. R., Delgado, J. D. y Otto, R. (2004). Canarias: ecología, medio ambiente y desarrollo. Las Palmas de Gran Canaria. Gobierno de Canarias, Consejería de Política Territorial y Medio Ambiente.

Flores, H. E., Martínez, M., Oropeza, J. L., Mejía, E. y Carrillo, R. (2003). Integración de las EUPS a un SIG para estimar la erosión hídrica del suelo en una cuenca hidrográfica de Teatitlán, Jalisco, México. Terra Latinamericana,21,2, p. 233-244.

García-Ruiz, J. M., Lasanta, T. y Sobrón, I. (1988). Problemas de evolución geomorfológica en campos abandonados: El Valle del Jubera (Sistema Ibérico), Zubía, 6, p.99-114.

García-Ruiz, J. M. y López-Bermúdez, F. (2009). La erosión del suelo en España. Zaragoza: Sociedad Española de Geomorfología (SEG), 441 pág. Zaragoza.

Gaspari, F. J., Delgado, M. I. y Senisterra, G. E. (2009a). Simulación espacio-temporal de la erosión hídrica superficial en una cuenca serraba bonaerense. Argentina. GeoFocus,9, p. 67-82.

Gaspari, F. J.; Delgado, M. I. y Denegri, G. A. (2009b). Estimación espacial, temporal y económica de la pérdida de suelo por erosión hídrica superficial. Terra Latinoamericana, 27, 1, p. 43-51.

Geler, T., Ligtenberg, A y Rolf, A. (2004). Modelo para el pronóstico de la dinámica de erosión en los suelos debido a los cambios en el uso de la tierra. Mapping, (94), 32-51 
Grimm, M., Jones, R. J. A., Rusco, E. y Montanarella, L. (2003). Soil erosion risk in Italy: a revised USLE approach.

European Soil Bureau Research Report No.11, EUR 20677 $E N$, (2002). Office for official publications of the European communities, Luxembourg, 28 p.

Hernández-Calvento, L. (1996). Modelización espacial del riesgo de erosión de suelos mediante SIG. Aplicación al estudio de la extracción de suelos en Gran Canaria (Memoria de Licenciatura). Universidad de Alcalá de Henares.

Hernández-Calvento, L., Romero, L., Ruiz, P. y PérezChacón, E. (1998). El riesgo de erosión como factor limitante de las extracciones de suelo en Gran Canaria. Una aproximación mediante SIG", en Gómez Ortiz, A. y Salvador Franch, F. (eds.). Investigaciones recientes de la Geomorfología española. Barcelona, p. 631-640.

Hernández-Rodríguez, O., Mongil Manso, J., Díaz Gutiérrez, V. y Navarro Hevia, J. (2014). Evolución de la erosión hídrica en la parte media y alta de la cuenca del río Corneja (Ávila, España). XII Congreso Nacional del Medio Ambiente, Madrid, 13 p.

IRYDA (1981). Estudio de los suelos regables de la isla de Gran Canaria Instituto de Reforma y Desarrollo Agrario (inédito).

IWG, W. (2007): World reference base for soil resources 2006, first update 2007. FAO, Roma.

Jager, S. (1994). Modelling regional soil erosion susceptability using Universal Loss Equation and GIS. First International Congress of the European Society for Soil Conservation, p. 161-177.

Jain, M. K. y Das, D. (2010). Estimation of Sediment Yield and Areas of Soil Erosion and Deposition for Watershed Prioritization using GIS and Remote Sensing. Water. Resour. Manage, 24, p. 2091-2112.

Jongman, R. H. G. (2002). Homogenisation and fragmentation of the European landscape: ecological consequences and solutions. Landscape and Urban Planning, 58, p. 211-221.

Kouli, M., Soupios, P. y Vallianatos, F. (2009). Soil erosion prediction using the Revised Universal Soil Loss Equation RUSLE) in a GIS framework, Chania, Northwestern Crete, Greece. Environ. Geol., 57, p.483-497.

Lahlaoi, H., Rhinane, H, Hilali, A., Lahssini, S. y Khalile, L. (2015). Potential erosion risk calculation using remote sensing and GIS in Oued El Maleh watershed, Morocco. Journal of Geographic Information System, 7, 128-139.

Lasanta, T., Vicente-Serrano, S. y Cuadrat, J. M. (2000). Marginación productiva y recuperación de la cubierta vegetal en el Pirineo: un caso de estudio en el Valle de Borau. Boletín de la A.G.E., 29, p.5-28.
Lasanta, T. (2010). Evolución regional y dinámica del paisaje en La Rioja (1950-2010). Zubía, 28, p. 49-88.

Lee, S. (2004). Soil erosion assessment and its verification using the Universal Soil Loss Equation and geographic information system: a case study at Boun, Korea. Environ. Geol., 45, p. 457-465

Lu D, L1 G, Valladares G. S., Batistella, M. (2004). Mapping soil erosion risk in Rondonia, Brazilian Amazonia: Using RUSLE, remote sensing and GIS. Land. Degrad. Dev., 15, p. 499-512.

Ma, J. W. y Xue, Y. corresponding author, C. F. Ma \& Z. G. Wang (2003). A data fusion approach for soil erosion monitoring in the Upper Yangtze River Basin of China based on Universal Soil Loss Equation (USLE) model, International Journal of Remote Sensing, 24:23, 4777-4789.

McCool, D. K., Foster, G. R.; Mutchler, C. K. y Meyer, L. D. (1989). Revised slope length factor for the universal soil loss equation, TRANSACTIONS of the ASAE, 32(5), p. 1571-1576.

Martin A., Gunter J. y Regens J. (2003). Estimating erosion in a riverine watershed, Bayou LibertyTchefuncta River in Louisiana. Environ. Sci. Pollut. Res., 4, p. 245-250.

Montes, M., Domínguez, M y Ventura, E. (2001). Metodología para la estimación del riesgo de erosión hídrica en cuencas hidrográficas utilizando un SIG. http://www.bvsde.paho.org/bvsaidis/impactos/ mexicona/R-0233.pdf.

Mitasova, H., Brown, W. M., Johnston, D., Mitas, L. (1996). GIS tools for erosion/deposition modeling and multidimensional visualization. PART II: Unit Stream Power-Based Erosion/Deposition Modeling and Enhanced Dynamic Visualization. Report for USA CERL. University of Illinois. Urbana-Champaign, IL. 38 p.

Orúe, M. E., Laterra, P. y Cabria, F. (2007). Expansión de la frontera agrícola en Argentina y erosion hídrica: mapas de riesgo utilizando el Modelo USLE con apoyo de SIG. TELEDETECCIÓN_Hacia -un mayor entendimiento de la dinámica global y regional. Ed. Martin.

Ozcan, A. U., Erpul, G., Basaran, M. y Erdogan, H.E. (2008). Use of USLE/GIS technology integrated with geostatistics to assess soil erosion risk in different land uses of Indagi Mountain Pass-Cankiri, Turkey. Environ. Geol., 53, pp.1731-1741.

Padrón, P.A.; González, M.C., Hernández, L. A., Jiménez, C. C. y Ortega, M. J. (1991). Erosividad de las lluvias en las Islas Canarias Occidentales; XVIII Reunión Nacional de Suelos, Dpto. de Edafología y Geología, Universidad de La Laguna, pp. 459-470. 
Panagapoulos, T. Cakula, A. y Ferreira, V. (2011). Soil erosion risk assessment at the watershed of Alqueva Dam, Portugal. Protection and restoration of the environment XI. Environmental geotechnology, pp.1318-1327.

Pandey, A., Chowdary, V. M. y Mal, B. C. (2007). Identification of critical erosion prone areas in the small agricultural watershed using USLE, GIS and remote sensing, Water Resour. Manage., 21, p.729-746

Reis, A. R., Lourenço, J. M. M., Parker, A. y Alencoao, A. (2014). Estimative of sediment yield in mountainous catchments: a pilot study in a meso-scale catchment of the Douro Basin, Northern Portugal. Comunicacoes Geológicas 101, Especial II, pp. 1.051-1.054.

Renard, K. G., Foster, G. R., Weesies, G. A. y Porter, J. P. (1991). Revised Universal Soil Loss Equation. J. of Soil andWater Conservation, 46, p. 30-33.

Rodríguez Rodríguez, A. (2002). Erosión y desertificación. Naturaleza de las Islas Canarias. Ecología y Conservación. Editorial Turquesa, Santa Cruz de Tenerife, p. 320-320.

Rodríguez, M. F., Florentino, A., Gallardo, J. y García, R. A. (2004). Sistemas de Información Geográfica en la evaluación de la erosión hídrica en Badajoz-España aplicando la metodología USLE. Agronomía Tropical, 54 (4), p. 391-409

Rojas-González, A. M. (2008). Soil erosion calculation using remote sensing and GIS in Río Grande de Arecibo watershed, Puerto Rico. ASPRS. Annual Conference, p.6.

Romero, L. E. (2015). Consecuencias geomorfológicas del abandono agrícola en la cuenca del Guiniguada (Gran Canaria, Islas Canarias): aplicación a la conservación del patrimonio de bancales. Tesis Doctoral (Inédita), 676 paginas. Universidad de Las Palmas de Gran Canaria.

Romero, L. E., Pérez-Chacón, E., García, L. y HernándezCordero, A. (2015). Análisis de la evolución (19602002) del paisaje agrícola mediante SIG: aplicación a la cuenca del Guiniguada (Gran Canaria, Islas Canarias, España). En: De la Riva, J., Ibarra, P., Montorio, R., Rodrígues, M. (Eds.) Análisis espacial y representación geográfica: innovación y aplicación, p. 1.203-1.209. Universidad de Zaragoza-AGE.

Schmidt, B. L., Allmaras, R. R., Mannering, J. V. y Papendick, R. I. (1982). Determinants of soil loss tolerance.

American Society of Agronomy - Soil Science Society of America. Madison, 153 p.

Sánchez, J. (1975). Características y distribución de los suelos de la isla de Gran Canaria. Tesis Doctoral (Inédita).Universidad de La Laguna.
Sánchez, J., Ríos, C., Pérez-Chacón, E. y Suárez, C. (1995). Cartografía del potencial del medio natural de GranCanaria. Cabildo Insular de Gran Canaria. Las Palmas de Gran Canaria, 165 p. y 7 mapas.

Sánchez-Bosch, I. y Martínez- Casanovas, J. A. (2000). Influence of land use changes land traditional soil conservation practices on erosion in the Pendès vineyard región (Catalonia). Abstracts book of the Third International Congress of the European Society for Soil Conservation: Man and Soil at the Thrid Millenium. Valencia.

Seeger, M. y Beguería, S. (2003). La respuesta hidrológica en dos cuencas experimentales con diferentes usos del suelo en el Pirineo aragonés. En: Marzzolf, I., Ries, J. B., De La Riva, J. y Seeger, M. (Eds.) El cambio en el uso del suelo y la degradación del territorio en España. p. 203-221. Zaragoza. Johann Wolfgang Goethe- Universität Frankfurt am Main y Universidad de Zaragoza.

Strahler, A. (1964). Quantitative geomorphology of drainage basins and cannel networks. En: Chow, V. T. Handbook of Applied Hydrology, 4-39/4-76. McGrawHill, New York.

Torres-Quevedo García De Quesada, M., Jarabo Sánchez, F., del Palacio Fernández-Montes, E., Garcia De Las Barreras, J. A. (2004). El Inventario Nacional de Erosión de Suelos 2002-2012, Revista Montes, 75, p. 34-41.

Vaudour, J. y Bonin, G. (1991).Terrasses de culture: leur evolution après abandon et mode de gestion minimum. Programme E.G.P.N. Consequences de la déprise agricole. Documento de síntesis, 19 pp., más apéndices.

Van Rompaey, A. J. J., Verstraeten, G., Van Oost, K., Govers, G. y Poesen, J. (2000). Modelling mean annual sediment yields using a distributed approach. Earth Surface Processes and Landforms, 26, pp. 1221-1236.

Van der Knijff, J. M., Jones, R. J. A. y Montanarella, L. (2000). Soil Erosion Risk Assessment in Europe, EUR 19044 EN, 34pp.

Williams, I.R. (1975). Sediment yield predictioo with universal equation using runoff energy factor. United States Department of Agriculture, Agricultural Research Service S-40, pp. 244-252.

Wischmeier, W.H. y Smith, D.D. (1978). Predicting rainfall erosion losses. A guide to conservation planning. U.S. Department of Agriculture, Agricultura Hanbook, 537, p.58.

Yang, D., Kanae, S., Oki, T., Koike, T. Y Musiake, K. (2003). Global potential soil erosion with reference to land use and climate changes. Hydrological Processes, 17, pp. 2913-2928. 